\title{
Development of Autonomous Magnetometer Rotorcraft for Wide Area \\ Assessment
}

Roelof Versteeg

Matt Anderson

Les Beard

Eric Corban

Darryl Curley

Jeff Gamey

Ross Johnson

Dwight Junkin

Mark McKay

Jared Salzmann

Mikhail Tchernychev

Suraj Unnikrishnan

Scott Vinson

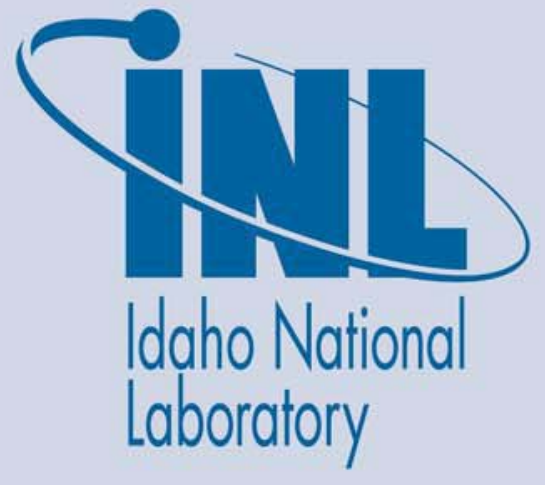

\section{April 2010}

The INL is a U.S. Department of Energy National Laboratory operated by Battelle Energy Alliance 
INL/EXT-10-18291

\title{
Development of Autonomous Magnetometer Rotorcraft for Wide Area Assessment
}

\author{
Roelof Versteeg \\ Matt Anderson \\ Les Beard \\ Eric Corban \\ Darryl Curley \\ Jeff Gamey \\ Ross Johnson \\ Dwight Junkin \\ Mark McKay \\ Jared Salzmann \\ Mikhail Tchernychev \\ Suraj Unnikrishnan \\ Scott Vinson
}

April 2010

\section{Idaho National Laboratory \\ Idaho Falls, Idaho 83415}

http://www.inl.gov

Prepared for the

U.S. Department of Defense

Under SERDP SEED Award 1509:2206 and for the

U.S. Department of Energy

Under DOE Idaho Operations Office

Contract DE-AC07-05ID14517 


\section{Table of contents}

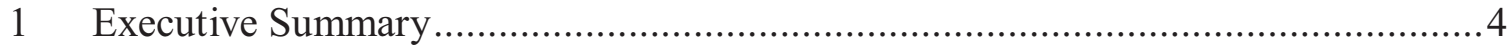

2 Summary of previous results.................................................................. 7

2.1 Background: Helicopter based UXO detection ...................................... 7

2.2 Boundary conditions for the implementation of an effective UAV Helicopter

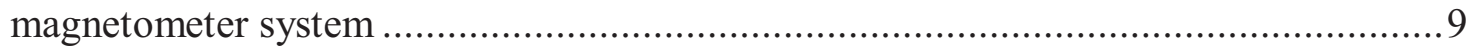

3 Applicability of UAV rotorcraft magnetometer systems to DoD UXO sites .......... 11

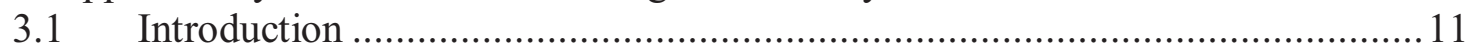

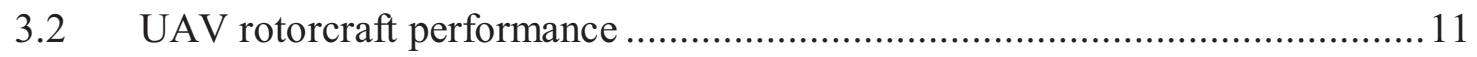

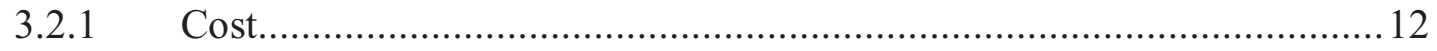

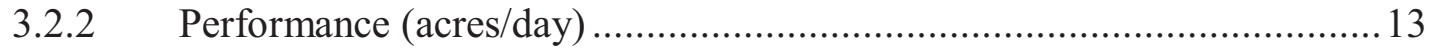

3.2.3 Performance (data quality) ........................................................... 15

$3.3 \quad$ Fieldsite applicability ...................................................................... 15

$4 \quad$ Feasibility of modular approach: component availability ............................... 18

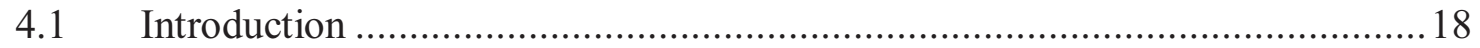

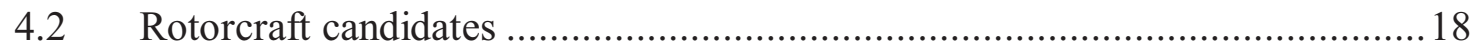

4.3 Autopilot/control laws availability for modular systems .......................... 21

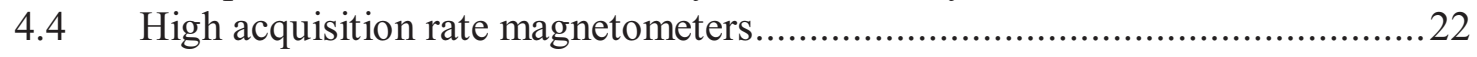

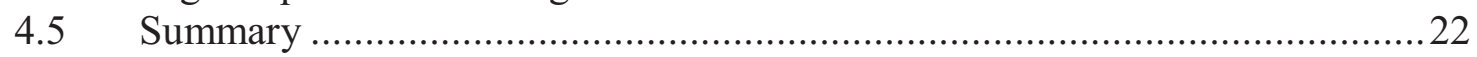

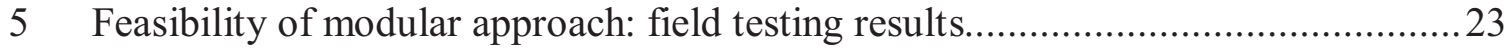

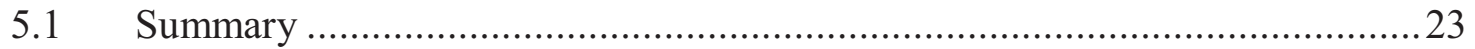

5.2 Field test 1: Assessment of terrain following capability and component

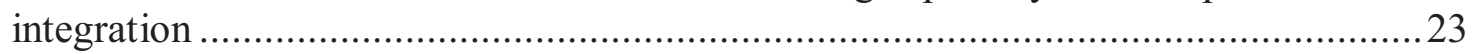

5.3 Field test 2: Assessment of ability to tune the controller to a boom and ability to collect high quality magnetic data from a modular helimag system .........................29

5.3.1 Mongoose test: assessment of ability to tune autopilot controller to boom 30

5.3.2 Mosquito test: magnetic data collection using a modular helimag system41

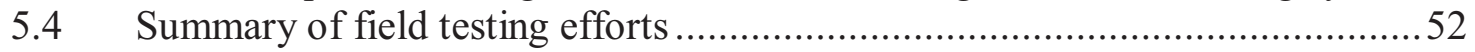

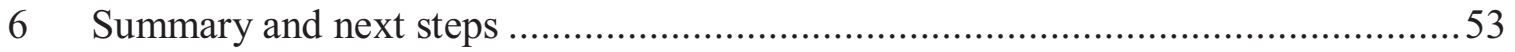

6.1 Summary and recommendation ..........................................................5

6.2 Research effort associated with developing prototype systems ....................53

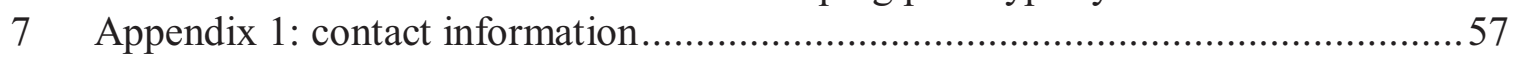

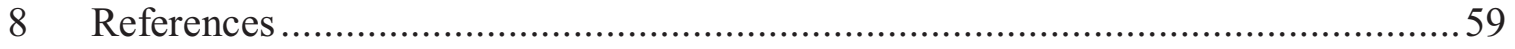




\section{Executive Summary}

Large areas across the United States are potentially contaminated with UXO, with some ranges encompassing tens to hundreds of thousands of acres. Technologies are needed which will allow for cost effective wide area scanning with 1) near $100 \%$ coverage and 2) near $100 \%$ detection of subsurface ordnance or features indicative of subsurface ordnance.

The current approach to wide area assessment is a multi-level one, in which medium altitude fixed wing optical imaging is used for an initial site assessment. This assessment is followed with low altitude manned helicopter based magnetometry. Subsequent to this wide area assessment targeted surface investigations are performed using either towed geophysical sensor arrays or man portable sensors. In order to be an effective tool for small UXO detection, the sensing altitude for magnetic site investigations needs to be on the order of $1-3$ meters. These altitude requirements mean that manned helicopter surveys will generally only be feasible in large, open and relatively flat terrains. While such surveys are effective in mapping large areas relatively fast there are substantial mobilization/demobilization, staffing and equipment costs associated with these surveys (resulting in costs of approximately $\$ 100-\$ 150 /$ acre). In addition, due to the low altitude there are substantial risks to pilots and equipment. Surface towed arrays provide high resolution maps but have other limitations, e.g. in their ability to navigate rough terrain effectively.

There is thus a need for other systems which can be used for effective data collection. An UAV (Unmanned Aerial Vehicle) magnetometer platform is an obvious alternative. The motivation behind such a system is that it reduces risk to operators, is lower in initial and Operational and Maintenance (O\&M) costs (and can thus potentially be applied to smaller sites) and has the potential of being more effective in terms of detection and possibly characterization (through the use of dynamic acquisition, i.e. survey mission inflight reprioritization).

However, while UAV data acquisition from fixed wing platforms for large ( $>200$ feet) stand off distances is relatively straight forward, a host of challenges exist for low standoff distance ( $\sim 6$ feet) UAV geophysical data acquisition. In a previous phase of this effort (reported in (Versteeg, McKay et al. 2007)) the primary challenges associated with a low stand off distance autonomous UAV magnetometer platform were identified and investigated. The conclusion of this previous phase was that the challenges associated with construction of an effective autonomous UAV magnetometer platform could be resolved successfully and that construction of a successful autonomous UAV magnetometer platform was feasible at least in theory.

However, at the completion of this initial effort several open questions remained which precluded the full scale development of systems. These were 
- Given their expected performance and cost, are UAV rotorcraft magnetometer applicable to and relevant for DoD sites and if so, what are the general attributes of such systems in terms of sensor arrays, flying time, cost and performance

- In light of the expected answer to the first question (that multiple systems which would provide a suite of different capabilities which could be applied to a range of terrains), what approach should be taken in the design and construction of such systems?

The second question was motivated by a discussion between the PI and the SERDP office on what approach has the highest likelihood of success in creating fieldable systems which meet DoD needs. Two different approaches exist:

1. Approach 1: a "closed system" approach. In this approach a UAV magnetometer platform would be created which is built on top of an existing integrated UAV platform in which rotorcraft, autopilot and groundstation are all tightly integrated by one manufacturer. One would then work with the manufacturer to integrate magnetometers and auxiliary sensors on such platforms. An example of such an approach would be one in which the Yamaha system would be used as a base platform.

2. Approach 2: A "modular system" approach. In this approach a UAV magnetometer platform would be created which uses existing components ( $\mathrm{RC}$ controllable rotorcraft, autopilots, magnetometers) and integrates them into systems.

In 2006 the project team initially advocated the first approach. However, since then it became clear that this approach has several weaknesses: it ties one in with a specific platform and manufacturer and the ability to do extensive modifications to such platforms (which are required to mount booms) looks to be limited. There is also some risk that the selected manufacturer may discontinue sale or development of the selected system. At the same time advances in autonomous rotorcraft efforts seemed to increasingly enable the modular approach.

This then led to the effort on which we report here: a one year effort with as primary goal to provide a recommendation to SERDP for a path forward in the implementation of one or more autonomous unmanned magnetometer rotorcraft platforms. This recommendation (which is provided in chapter 6) is based on the following three elements

a) An assessment on the applicability of autonomous rotorcraft magnetometer systems to the current DoD site inventory, and an initial assessment of which type(s) of autonomous unmanned magnetometer rotorcraft platforms (in terms of performance characteristics such as payload, altitude, obstacle avoidance, production rate and flight time) would be most relevant to this inventory (chapter $3)$;

b) An evaluation of the feasibility of assembling such platforms from commercial components (unmanned rotorcraft, control systems and sensors - both 
magnetometer sensors and supporting sensors). This evaluation included several highly successful field tests (chapter 4 and 5);

c) A recommendation of the path forward, which includes a detailed outline of the efforts required in the design, assembly and testing of different modular platforms (chapter 6)

Note that in addition to the chapters reporting on each of these elements, a brief background on the challenges associated with autonomous UAV rotorcraft magnetometer systems is provided in chapter 2 . 


\section{Summary of previous results}

\subsection{Background: Helicopter based UXO detection}

The use of helicopter based UXO detection using magnetic sensors started in the US in 1994. Efforts in this area have resulted in a relatively mature technology. There is agreement about the best design (see the work of (Gamey and Mahler 1999) who concluded that a mounted sensor performed significantly better than towed sensors). There are also increasingly sophisticated acquisition systems which collect data from multiple magnetometers at high sampling rates $(\sim 100 \mathrm{~Hz})$, and associated with these acquisition systems are highly accurate positioning systems and sophisticated semi automated data interpretation schemes (Salem, Gamey et al. 2001). A good review of these efforts can be found in (Doll, Gamey et al. 2001).

Two helicopter based systems have been developed over the past years in the USA, the ORAGS (Oak Ridge Airborne Geophysical System) systems developed by Oakridge National Lab (Doll, Gamey et al. 2001; Gamey, Doll et al. 2003) and currently operated by Battelle and the MTADS (Multi-sensor Towed Array Detection System) system developed by the Naval Research Laboratory (Nelson, Wright et al. 2004) which was operated by Sky Research. Recently Sky Research has developed a new system (Billings and Wright, 2009) which improves on the original ORNL system in several facets. All systems are similar in design (figure 2.1), consisting of a boom equipped with a number of cesium vapor magnetometers mounted in the front of a manned helicopter. The ORNL system has 8 magnetometers mounted at 1.75 meter spacing, while the NRL system has 7 magnetometers mounted at 1.5 meter spacing. Note that several versions of the ORNL have been developed over the years with different configurations (e.g. the Hammerhead Array (Doll, Gamey et al. 2001) and the Vgrad system (Gamey, Doll et al. 2003)). 

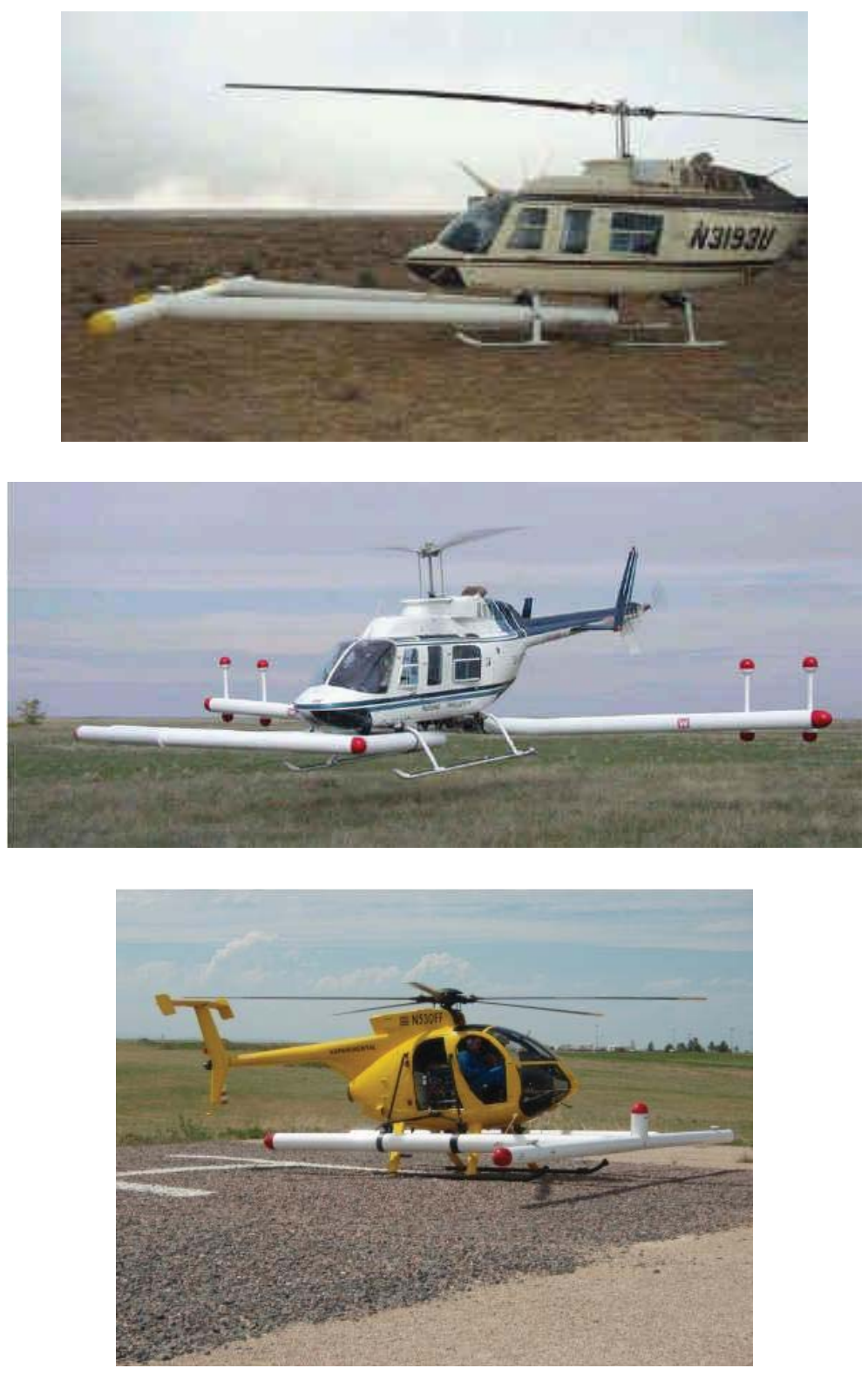

Figure 2.1 Original MTADS Systems from NRL (top)(Nelson, Wright et al. 2004) ,ORAGS-VGrad system from ORNL (middle) (Gamey, Doll et al. 2003) and new Sky Research system (Billings and Wright, 2009)

These systems have successfully been used to map and characterize UXO distributions on several sites including the Badlands Bombing Range in 2000 (Doll, Gamey et al. 2001), and Isleta Pueblo (Albuquerque, NM) in 2003 (Nelson, Wright et al. 2004). They have demonstrated the capability to assess open, flat terrain and detect large individual ordnance items in locations where sensor height can be maintained at very low above ground altitudes (1-2 meters).

The performance of both of these systems is relatively similar: they fly at between 1 and 3 meters above the ground surface, they fly at speeds of about 10-20 meters a second, 
they collect data from each sensor at about $60-100 \mathrm{~Hz}$, and they both collect a swath of data at a time. The area covered by these systems is in the order of 30-50 acres/hour. Positioning of the sensor data is provided by RTK DGPS, with a reported accuracy of post acquisition positioning of each sensor of $5 \mathrm{~cm}$ and a conformance to a predefined flight path on the order of a meter. However, these systems have several characteristics which have prevented their broad application to many sites contaminated with UXO. These characteristics include:

(1) System and O\&M cost. These include both the cost of the initial system (put by (Nelson, Wright et al. 2004) at about $\$ 400 \mathrm{~K}$ ) and the cost of system deployment and operating costs of between $\$ 100$ and $\$ 175$ per acre. A significant component of this cost is associated with the helicopter and the pilot, and the need to have onsite geophysical experts for data interpretation. Extrapolation of this cost to the 50 million acres yields a total assessment cost of several billion dollars.

(2) Site limitations. The need to fly low is imposed by the need to detect UXO signatures. However, safety considerations only allow this for open, flat areas. In sites with significant topography (or even gently undulating terrain) these systems can not maintain the 1-2 meter altitude necessary for good UXO mapping.

(3) Limitations in ability to perform detailed localized mapping. The current systems are limited in their ability to detect small ordnance, and cannot "zoom in" on specific areas of interests (also due to limitations in absolute positioning). Thus, these systems do not provide the required ability for $100 \%$ mapping and require additional land based surveys.

While future work on these systems (including addition of electromagnetic sensors) is expected to provide incremental enhancements to these systems, the primary characteristics listed above are not likely to change substantially. The premise underlying our efforts is that the current limitations of airborne magnetometry can be overcome by replacing the current manned helicopter platform by an autonomous UAV helicopter platform. In the remainder of this section we assess the primary challenges associated with such an autonomous UAV helicopter platform

\subsection{Boundary conditions for the implementation of an effective UAV Helicopter magnetometer system}

An ideal UAV Helicopter magnetometer system should allow for the effective and high confidence detection and discrimination of munitions of concern. This should be done by the cost effective and safe acquisition of regularly spaced values of the magnetic field and/or of the gradient of the magnetic field as closely as possible to the munitions of concern (the benefits of proximity for both identification and characterization are well known (Stanley 2003)). Implementation of an UAV rotorcraft magnetometer system is conditional on the following three main conditions 
1. The commercial availability of helicopter UAVs which can operate autonomously and can carry a payload required for data collection, positioning, obstacle avoidance and communication for appropriate flight times.

2. The feasibility of integrating collision avoidance hardware and software in such a manner that a helicopter UAV can fly at elevations of about 1.5 meters at speeds of about two to ten meters per second.

3. The feasibility of collecting high quality magnetic data on such a platform.

As described in (Versteeg, McKay et al. 2007) all of these conditions can be met in theory. This report discusses to what extent they can be met in practice. 


\section{Applicability of UAV rotorcraft magnetometer systems to DoD UXO sites}

\subsection{Introduction}

In order for UAV rotorcraft systems to be applicable and relevant to DoD UXO sites two conditions must be met:

1. These systems must either allow for acquisition where current sensing modalities are not feasible or provide a benefit in either cost, performance or data quality over these existing modes.

2. The expected performance envelope of these systems must map to a substantial percentage of DoD sites

This chapter addresses these two conditions. First, the expected performance of fieldable systems is addressed. Second, based on this performance and a database of DoD UXO sites we analyze whether these systems would be relevant to a substantial percentage of DoD sites.

\subsection{UAV rotorcraft performance}

There is a significant experience within both DoD and a number of commercial companies on the cost, performance and data quality associated with the three primary modes of geophysical data collection at MEC sites: manually carried sensors, towed systems/carts and manned helicopter systems. In order for a UAV rotorcraft system to be relevant for site characterization, it would need to either allow for acquisition where current sensing modalities are not feasible or provide a benefit in either cost, performance or data quality over these existing modes.

It is thus important to formulate an initial quantitative expectation on cost, performance and data quality for an UAV rotorcraft system which can be compared against existing sensing modalities. While such an expectation will obviously have large error bars (which will be reduced over time), it serves two important objectives. First, it provides a first insight to the funding agency and end users on what such a system will do (and allows them to provide constructive input in the development process). Second, such an expectation will be used to guide rotorcraft choice, the number and configuration of magnetometers as well as the performance specifications of one or more prototype systems.

Note that we currently exclude in this discussion any consideration of the cost of post processing of the data, as well as any consideration on the safety benefit associated with flying unmanned vs manned rotorcraft. 


\subsubsection{Cost}

The cost of deploying a system can be separated in

1. depreciation cost

2. mobilization and demobilization cost

3. operational costs

The depreciation cost is a function of the initial system cost, the effective life of the system and the amount of use of a system. The initial system cost will obviously depend strongly on the component choice of the system. Some components will need to be present in all systems (e.g. autopilot, differential GPS, altimeter, obstacle avoidance hardware, data acquisition system and ground station). The cost of the two other main components (the rotorcraft and magnetometers) will vary significantly. The cost of rotorcraft systems can range from several thousand dollars for the lowest cost systems to about $60-80 \mathrm{~K}$ for the upper end of the spectrum. Similarly, the magnetometer cost will be linear with the number of magnetometers, which could range from two to eight, and will also be dependent on the type of magnetometer selected. Based on what is currently known (and realizing that component costs are likely to change over the coming years) we feel that for an operational system produced in modest quantities the lowest achievable initial system cost would be about $\$ 120 \mathrm{~K}$ for a system with two magnetometers up to about $\$ 250 \mathrm{~K}$ for a system with eight magnetometers. Note however that with judicious design some or most of the components may be swappable between systems. The effective life of a system is estimated to be three years. This is likely to be conservative and some components are likely to have a longer lifespan. However, based on this (and ignoring some other factors), a yearly depreciation cost will be around 40 to $80 \mathrm{~K}$. If a system sees use for 60 days a year, the depreciation cost would be some 600 to $1200 \$$ a day. Of course, higher use rates would decrease this cost proportionally.

The mobilization costs are dependent on the size of the system. Several of the rotorcraft candidates can be shipped by commercial carriers at modest costs (few hundred \$). Larger systems (such as the Mosquito, chapter 5), would require transportation in a dedicated trailer or flatbed, with mobilization costs dependent on the distance, but typically in the order of $\$ 2 \mathrm{~K}$.

The operational costs depend on the number of acres per day that such a system would cover, the number of operators (and the skill level of operators) required to operate this system, and the O\&M costs (mainly fuel). We consider here the operator cost. As discussed in the performance section below, we expect that systems like this would be able to cover between about 5 and 20 acres an hour (dependent on configuration, see section 3.2.2). As these systems are autonomous, they do not require licensed pilots (like manned helicopter systems require), and in theory operators would require only limited skillsets. Note that one of the issues which would need to be decided is whether operators need to be able to remotely pilot the rotorcraft (which is a non-trivial skill), or whether they are essentially there to push a stop button which would automatically abort the 
mission and retrieve the rotorcraft to base (for instance in the case of inclement weather or undetected obstacles). We currently estimate that we would need two operators at a burdened cost of $\$ 600 /$ day each.

Table 3.1 summarizes these costs for a five day effort in which a 250 acre site is covered (6 hr/day at about 8 acres/hr). This results in a cost per acre of about $\$ 64$.

\begin{tabular}{|l|r|l|r|}
\hline & Days & Unit cost & \\
\hline Mob cost & & $\$ 4,000.00$ & $\$ 4,000.00$ \\
\hline Operator cost & 5 & $\$ 1,200.00$ & $\$ 6,000.00$ \\
\hline Depreciation cost & 5 & $\$ 1,200.00$ & $\$ 6,000.00$ \\
\hline & & Total cost & $\mathbf{\$ 1 6 , 0 0 0 . 0 0}$ \\
\hline
\end{tabular}

Table 3.1 Cost for a five day effort for a 250 acre site.

It is obvious that the error bars on the cost (both of the components and the cost per acre) are substantial and intuitively this cost seems to be fairly low. However, even if the estimate is off by a factor of two it is still competitive with other efforts.

\subsubsection{Performance (acres/day)}

The performance of UAV rotorcraft systems is given by the combination of the system speed, the flight path spacing and the effective number of hours per day that such a system can fly. As in the cost section, the error bars on these numbers are currently substantial. In table 3.2 we give our best guess estimates for two different configurations using a path overlap of $25 \%$. Table 3.3 gives the acres/hr for the same parameters and configuration as shown in table 1 for different overlaps.

\begin{tabular}{|l|r|r|}
\hline & Configuration 1 & Configuration 2 \\
\hline Speed (m/s) & 5 & 10 \\
\hline Speed (km/hr) & 18 & 36 \\
\hline Speed (knots) & 9.7 & 19.4 \\
\hline Speed (miles/hr) & 11.2 & 22.4 \\
\hline Number of magnetometers & 3 & 7 \\
\hline Sensor spacing & 0.5 & 0.5 \\
\hline Array width & 1 & 3 \\
\hline Flight path spacing & 0.75 & 2.25 \\
\hline Flight path length/acre (in m) & 5461 & 1820 \\
\hline Time for 1 acre (seconds) & 1092 & 182 \\
\hline Acres/hr & 3.3 & 19.8 \\
\hline Acres/day (6 hr flighttime) & 20 & 119 \\
\hline
\end{tabular}

Table 3.2. Estimate for the performance of two different configurations with different speeds and number of magnetometers. A flightline overlap of $25 \%$ is used 


\begin{tabular}{|l|r|r|}
\hline & Configuration 1 & Configuration 2 \\
\hline No overlap & 4.3 & 26.3 \\
\hline $10 \%$ overlap & 4.0 & 23.7 \\
\hline $25 \%$ overlap & 3.3 & 19.8 \\
\hline $40 \%$ overlap & 2.7 & 15.8 \\
\hline $50 \%$ overlap & 2.2 & 13.2 \\
\hline
\end{tabular}

Table 3.3. Acres/hr for configurations shown in table 3.2 for different $\%$ overlaps between flight paths.

Table 3.2 has an underlying assumption on payload capability and assumes rotorcraft with a fairly decent payload capability (including fuel required for flight time). While the exact payloads for each configuration can vary based on the final component choice, configuration 1 will likely require at least a $40 \mathrm{lb}$ payload, and configuration 2 at least a $60 \mathrm{lb}$ payload. Of course, more payload translates in more fuel and flighttime.

It is obvious that by playing with the different numbers one can change the bottom line substantially. Arguments can be made to substantially change the numbers for each configuration. For instance, terrain topography and system responsiveness to obstacles will significantly impact the attainable speed

With current manned systems a flightline overlap of $25-40 \%$ is a typical number used in survey design and execution to provide confidence in the ability to get complete coverage. The amount of overlap required for autonomous rotorcraft helicopter to avoid coverage gaps will be dependent on a number of factors. These include the navigation accuracy of the combined sensor suite and navigation filter, latency in both the measurements and the control system, the speed of travel, the control power available and the bandwidth of control achieved on the helicopter, the helicopter's mass properties and aerodynamics, and the atmospheric conditions. Position accuracy of the current helicopter UAV navigation solution is bounded by the accuracy of the GPS solution employed. The best-available precision GPS equipment from Novatel (RT-2) that is already integrated with the autopilot is rated at $1 \mathrm{~cm}$ plus $1 \mathrm{ppm}$ horizontal position accuracy rms with a 20 $\mathrm{Hz}$ update rate.

One example of the possible gains which can be achieved by going from manual to automated systems can be found in automated farm equipment. In such equipment precision GPS is used to produce significant gains in efficiency by straightening lines of travel. This eliminates both gaps and unintentional overlap when compared to farm equipment that is manually piloted. Similar gains are expected from the automation of a helicopter. However, the helicopter will be more sensitive than ground equipment to disturbance from winds and gusts.

No attempt was made in the current control software to optimize the autopilot installation and control loops to achieve the best possible tracking of lines in the horizontal plane, and no tests have been performed that provide any meaningful measure 
of what level of tracking performance has or ultimately can be accomplished in this regard. Thus, for the purpose of generating initial estimates for use in this report, it is appropriate to employ the overlap requirement typically employed in manned helicopter operations (e.g. 25\%). However, it is anticipated that with the right combination of equipment and fine tuning, the required amount of overlap to ensure no gaps in sensor coverage when employing the unmanned helicopters could be significantly less than this.

In summary, the numbers in table 3.2 provide a likely operational performance range for unmanned autonomous systems of 3.3-20 acres/hr -substantially higher than ground based systems, but less than manned rotorcraft magnetometer systems.

\subsubsection{Performance (data quality)}

The data shown in chapter five shows that under good conditions a helicopter based system can collect datasets with comparable quality and noise to that of ground based datasets. Note that these data were collected at relatively slow speeds ( $\sim 3 \mathrm{~m} / \mathrm{second}$ ). This speed was mandated by the $20 \mathrm{~Hz}$ acquisition rate of the Geometrics G823A Cesium magnetometers used for the data acquisition, however it is likely that similar data quality would be attained at higher speeds.

We were able with these data to detect small targets such as individual golf ball sized submunitions (BLU 26s) and $60 \mathrm{~mm}$ mortar rounds. So long as the data quality is high, parameters such as magnetic dipole moment and orientation can be obtained through automated inversion methods, and these parameters can be used to adjust the ordering of a list of anomalies in terms of ordnance likelihood. Although magnetic data alone are usually insufficient for reliable discrimination in mixed ordnance sites, even a modest improvement in dig list ordering obtained through analysis of inversion results can cut field remediation costs. If discrimination becomes a high priority for autonomous survey platforms, one option is to add one a time or frequency domain electromagnetic (EM) system combined with a cued survey approach in which the unmanned helicopter can switch to a slow speed mode and collect high-density data around a suspected target using a hover mode. While this effort is outside the scope of the currently proposed follow up effort, the technologies required for autonomous rotorcraft magnetometer systems would provide a natural basis for autonomous rotorcraft EM systems.

\subsection{Fieldsite applicability}

Based on the results above we currently conceive of two different types of systems which can cover 20 to 100 acres per day. The question is then to what extent these systems would be applicable to the DoD site inventory. 
A database with the results of a recently completed survey of available FUDS site characteristics was provided by the SERDP office.. This database has information on 2514 sites, with for each site information on site location, site size, topography, vegetation and types of ordnance. It should be noted that this database does not break out any detail for large sites (in which there is likely to be a range of topographies and vegetation). Thus, the information in this database can only be used as an indicator.

The information in this database can be represented in multiple ways. For instance, we could look at the distribution of sites with specific acreages (figure 3.1) or at the total acreage distribution over sites (figure 3.2), at the vegetation distribution (Figure 3.3) or the topography distribution (Figure 3.4).

Figure 3.1 tells us that over $50 \%$ of sites have between 10 and 1000 acres. 1000 acres is typically considered the current lower limit of application of a manned helicopter airborne system but unmanned autonomous systems with the capabilities as discussed in section 3.2 would be well suited for surveys of these and smaller sites. While manned helicopter systems are currently likely to keep on dominating for surveys of large, flat sites, it is conceivable to apply autonomous rotorcraft systems to selected portions of large sites.

Figure 3.3 and 3.4 provide information on vegetation and topography. While it is unlikely in the near future that any airborne system would successfully fly between trees, it is more likely for a small, nimble autonomous system to be able to deal with gently rolling terrains and terrains with modest amount of vegetation.

In summary, while there are undoubtedly many sites and part of sites where autonomous rotorcraft magnetometer system would not be applicable, based on the current available information it seems likely that such systems would be applicable to at least $50 \%$ of the site inventory.

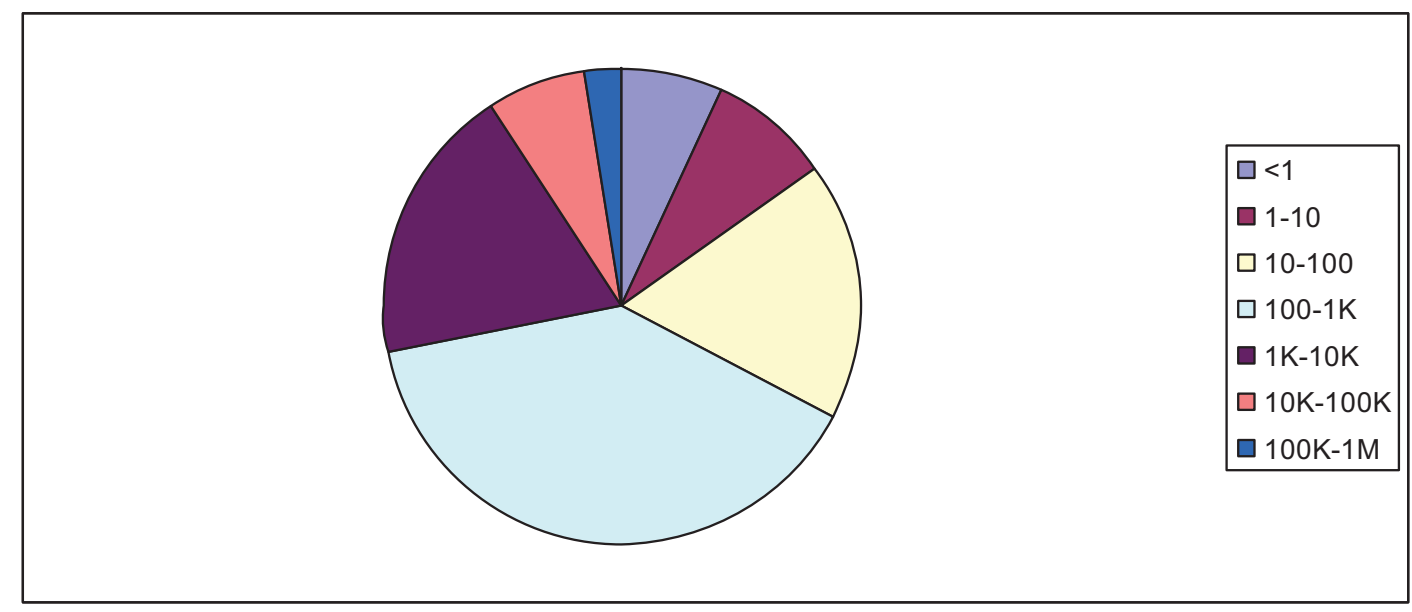


Figure $3.1 \%$ of sites with specific acreage

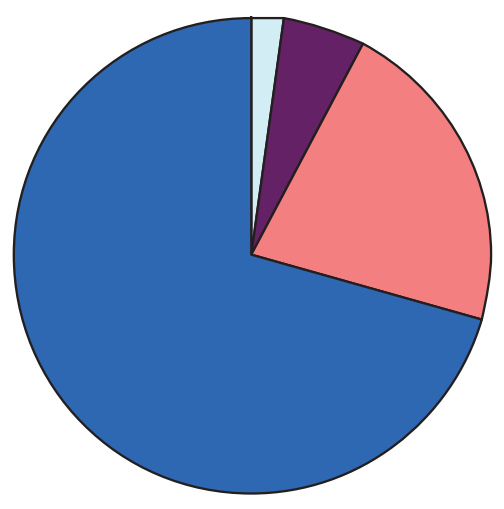

Figure 3.2 Distribution of acreage over sites.

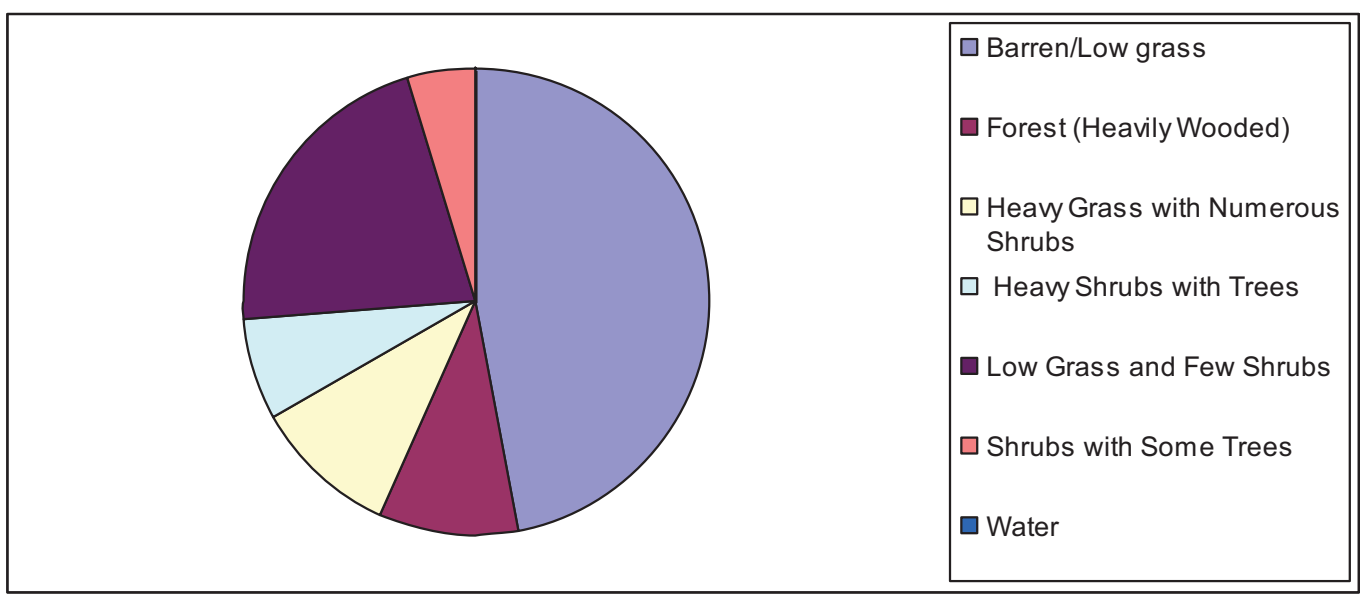

Figure 3.3 Distribution of vegetation over sites

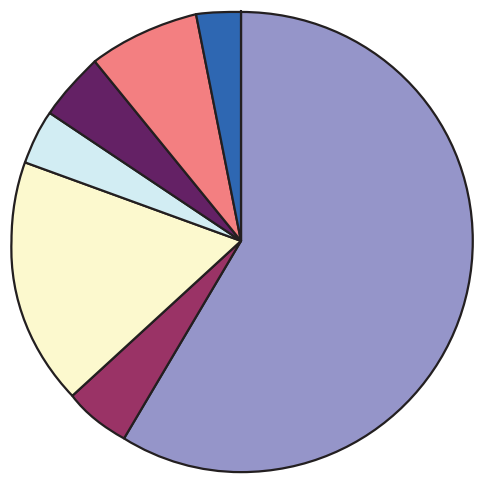

$\square$ Flat with Gorges or Gullies

$\square$ Gently Rolling

$\square$ Heavy Rolling

- Mountainous

$\square$ Rolling with Gorges or Gullies

$\square$ Water 
Figure 3.4 Distribution of topographies

\section{Feasibility of modular approach: component availability}

\subsection{Introduction}

The feasibility of a modular approach to the design of autonomous UAV rotorcraft magnetometer systems depends on the availability of mature components. In this chapter we discuss the status of the main critical components:

1. Rotorcraft which lend themselves to RC control/control by an autopilot

2. Rotorcraft autopilots and control laws

3. Magnetometers which can collect data at sufficient acquisition rates

Obviously there are a number of other components which are part of such systems (RTK DGPS, laser altimeters, communication hardware and so on). However, these components are essentially commodities: while it will be important to select components which have favorable characteristics in terms of power use, weight and accuracy, finding such components is relatively easy.

\subsection{Rotorcraft candidates}

A large number of rotorcraft exist which can be controlled by an autopilot (which typically uses the same control interface as used by a Remote Control (RC) interface. New and enhanced rotorcraft which integrate novel technologies and materials continue to come on the market and are used interchangeably for hobby purposes, commercial applications and R\&D. One way to consider rotorcraft candidates is by grouping them into five basic size categories;

1. Nano scale - systems less then $1 \mathrm{lb}$

2. Miniature $-1-5 \mathrm{lb}$

3. Small $-5 \mathrm{lb}$ to $501 \mathrm{~b}$

4. Midsized $-50-3001 b$

5. Large $-300+$

The Nano scale systems cannot carry much payload, perhaps a very small camera or an infared sensor, and are limited in operating range and flight duration. Miniature scale systems, while a little larger, can carry some bigger payloads but are still limited in range and capability and do not form realistic candidates. Therefore, our focus has been on the three size categories capable of deploying useful payload, that of a payload of $2 \mathrm{lb}$ and 
larger and a useful operating range. Note that a payload of $2 \mathrm{lbs}$ does not allow for a full fledged system, but is useful to investigate e.g. terrain following or obstacle avoidance abilities. As such abilities can be transferred between categories this allows development on one type of (cheap) hardware before deploying to more expensive hardware.

As mentioned in chapter 1, in the first phase of this effort we had focused on integrated systems such as the Yamaha Rmax or the Neural Robotic Autocopter (figures 4.1 and 4.2). However, in this phase we chose a modular approach. For this modular approach we thus only require the availability of RC controllable rotorcraft. Multiple such systems exist, including the Maxi Joker (Figure 4.3), the Mongoose (Figure 4.4) and (in the near future) the Mosquito (Figure 4.5) and the SiCK 70 (Figure 4.6).

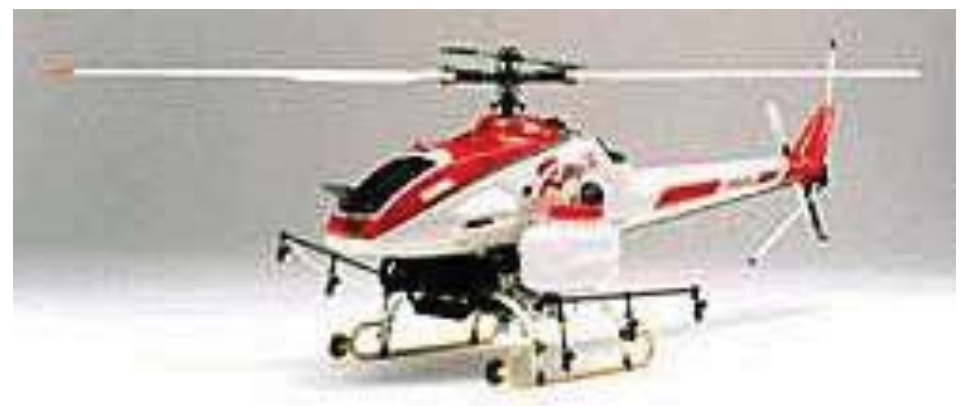

Figure 4.1. Yamaha RMAX

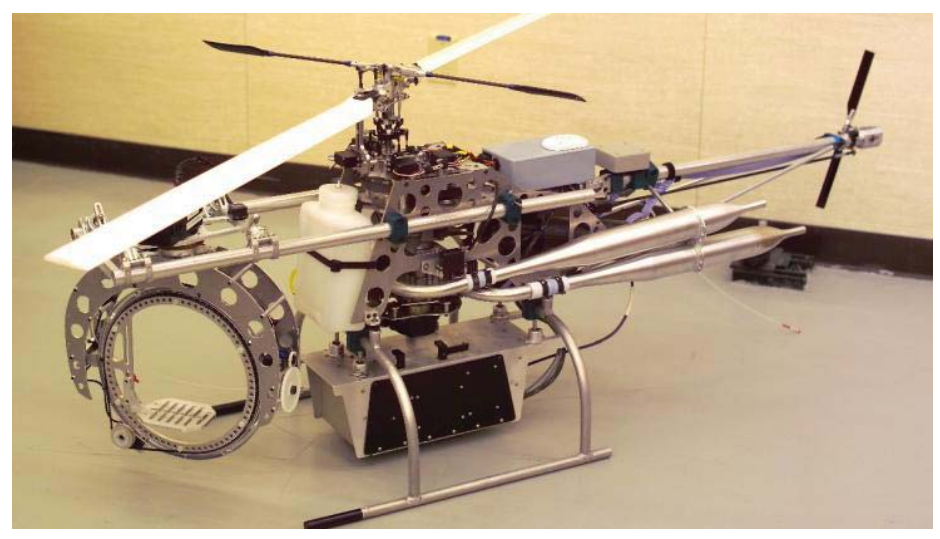

Figure 4.2 Neural Robotics Autocopter 


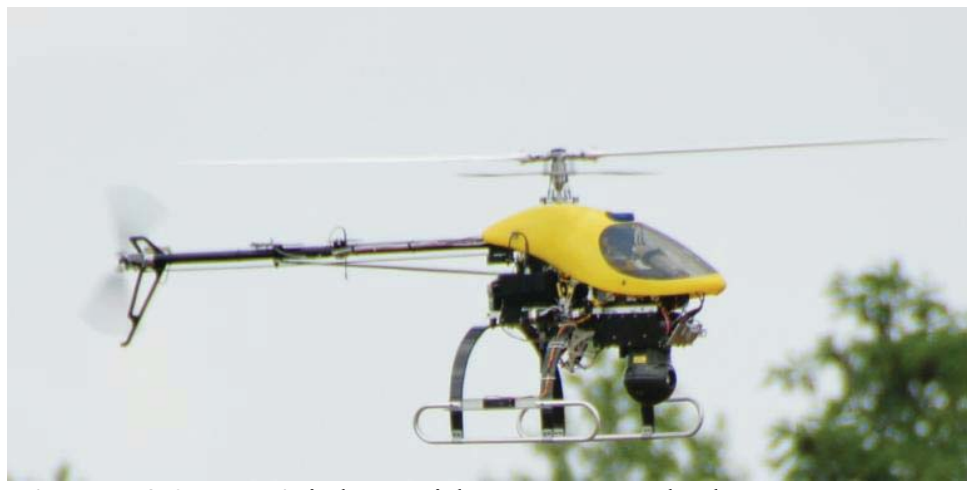

Figure 4.3 Maxi-joker with camera and Flux-gate Magnetometer

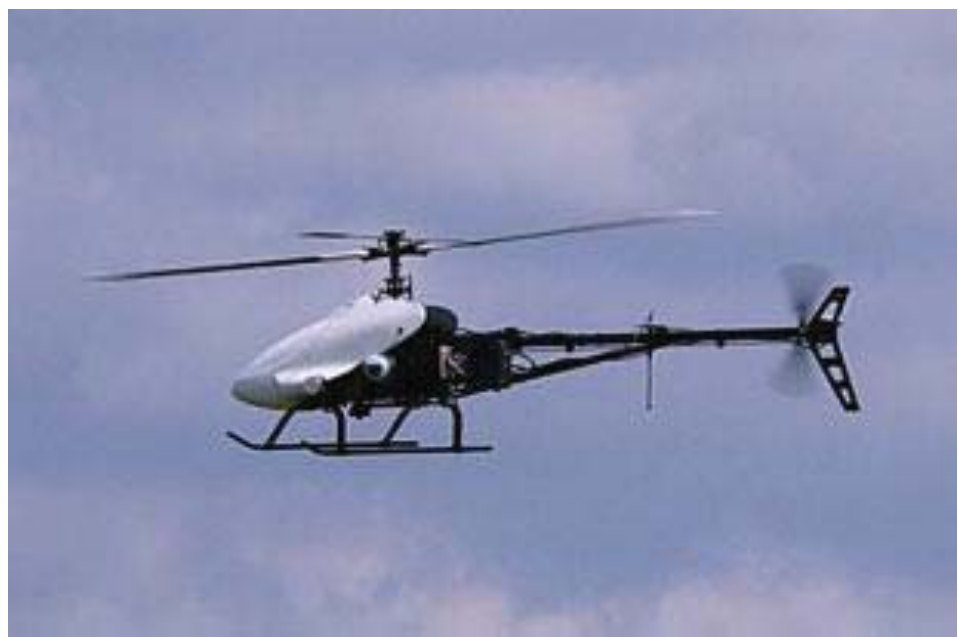

Figure 4.4 Mongoose (SICX 12)

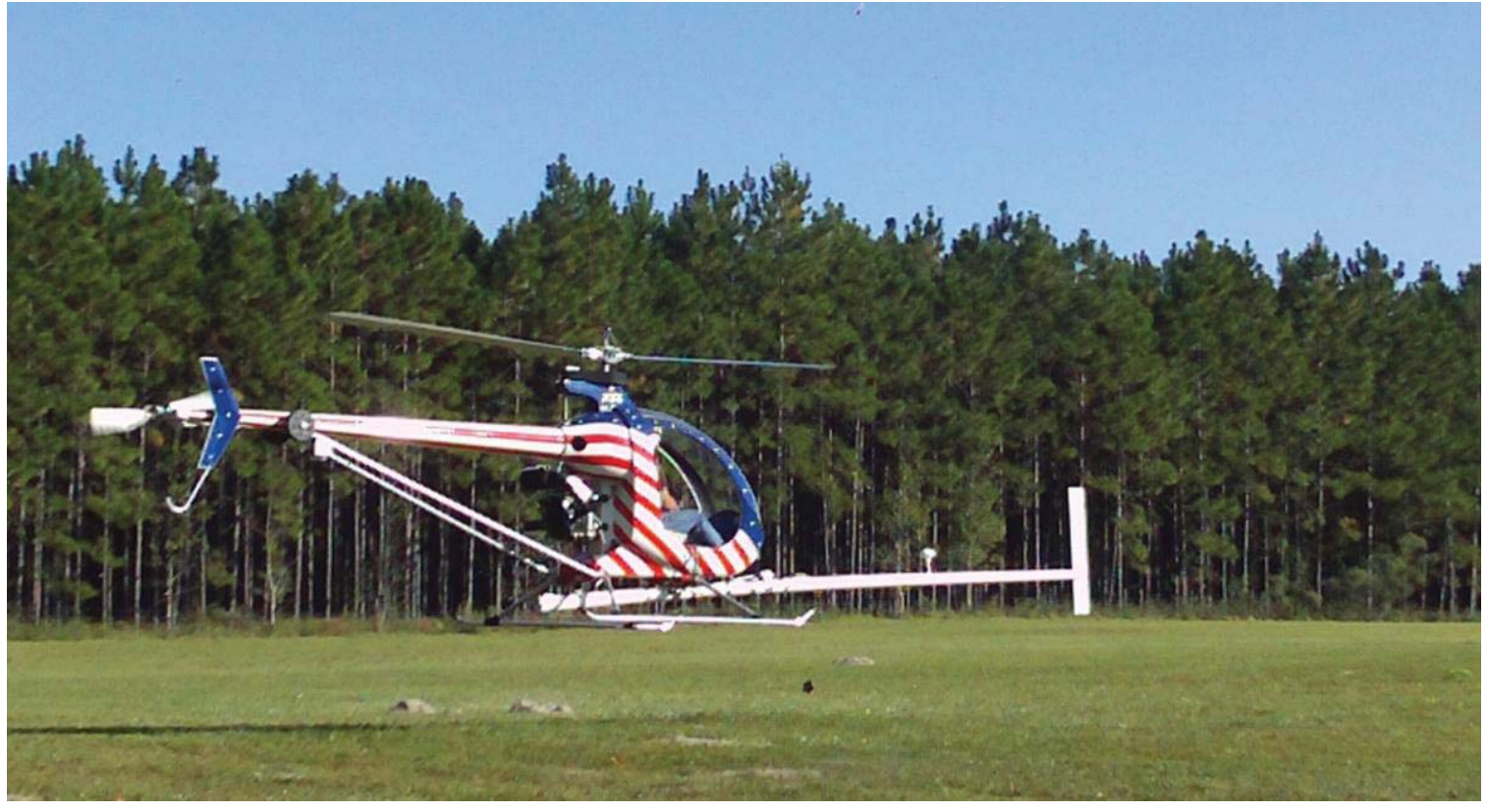

Figure 4.5 Mosquito. Note that this is a single seater, manned aircraft. However, efforts 
are currently underway to make this system remote controllable

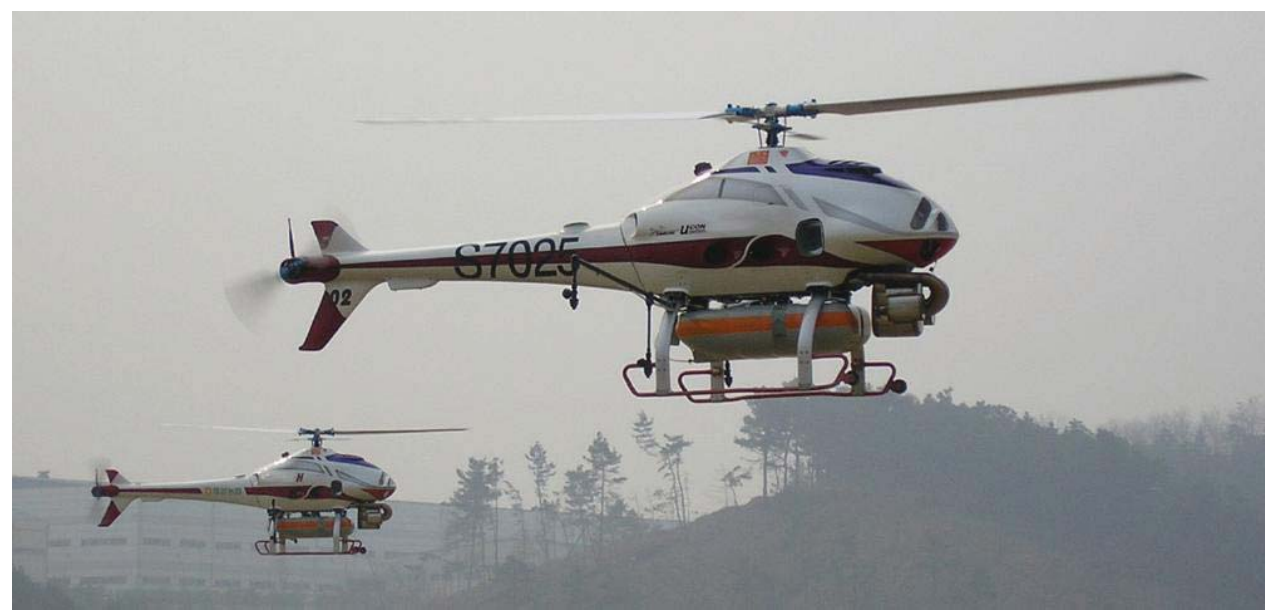

Figure 4.6 SiCX 70

In summary, there are currently many RC controllable rotorcraft in the small to large categories with payloads ranging from about $1 \mathrm{lb}$ (the Maxi Joker) to $250 \mathrm{lbs}$ (the Mosquito) which would meet our requirements, and new ones are becoming available periodically.. Or, the conclusion for RC controllable rotorcraft is that there are mature candidates available.

\subsection{Autopilot/control laws availability for modular systems}

Due to the complexity of controlling system stability, the first successes in autonomous flight of UAV helicopters only occurred in the late 1990s (Chapuis, Eck et al. 1997; MIT 1998). Since then several commercial platforms have been developed which offer autonomous flight capability (e.g. the Yamaha and the Neural Robotics platforms). These are so called "closed" systems in which the autopilot, the control software and the rotorcraft are tightly integrated. In fixed wing aircraft a number of commercial autopilots exist (see e.g. Chao et al, 2007 for an overview) which can relatively easily be integrated with a range of RC controllable RC aircraft. However, only recently have autopilots with generic rotorcraft capability become available. For our tests we selected an autopilot which was developed through a collaboration between Cloud Cap Technologies and Guided Systems. This controller is known as the Piccolo II with rotorcraft capability. It is a successor to the Piccolo controller which is used extensively in fixed wing UAV operations both by INL and other groups. The controller uses Guided Systems developed Neural Network control laws to control the rotorcraft, and by simple adjustment of the control laws to a specific rotorcraft the adaptive controller can control a broad range of rotorcraft. Specifically, the Piccolo II has successfully flown an electric powered Maxijoker (both without camera and with camera), a gas powered Mongoose, Logo, Leptron Agressor, a Vario XLC, and the Bergen Industrial Twin. The Piccolo will be flown on the Mosquito in the near future. 
While other similar modular autopilots with associated tuneable control laws are under development by other groups, the Piccolo II stands out with regard to the number of platforms it has successfully been deployed on. In addition (as discussed in chapter 5) the Piccolo II was demonstrated to be adaptive to substantial platform modifications (specifically, the addition of a boom). This demonstrated to the project team the flexibility of this controller and the associated control laws.

\subsection{High acquisition rate magnetometers}

One of the primary needs for airborne data acquisition is the availability of high quality magnetometers which can be sampled at sufficiently high sample rates. What "sufficiently high" means is still to be decided, but it will likely be in the order of several hundred Hz. For the effort discussed in chapter 5 Geometrics G823 A units were used with a maximum sample rate of $40 \mathrm{~Hz}$. However, Geometrics is coming out with novel magnetometers (table 4.1) which will be able to sample at significantly higher sample rates.

\begin{tabular}{|l|c|c|c|c|c|}
\hline Magnetometer & $\begin{array}{c}\text { Total } \\
\text { Weight (lbs) }\end{array}$ & $\begin{array}{c}\text { Sensor } \\
\text { size (in) }\end{array}$ & $\begin{array}{c}\text { Sensitivity } \\
(\mathrm{pT} / \mathrm{rtHz} \text { RMS }\end{array}$ & $\begin{array}{c}\text { Max Sample } \\
\text { Rate }\end{array}$ & $\begin{array}{c}\text { Power } \\
\text { Required }\end{array}$ \\
\hline G-823 & 4.4 & $\begin{array}{c}2.375 \text { dia } \\
\text { x 6 }\end{array}$ & 4 & 40 & $\begin{array}{c}\text { A start, } \\
500 \mathrm{ma}\end{array}$ \\
\hline $\begin{array}{l}\text { G-824 } \\
\text { (SupremaC) }\end{array}$ & 5 & $\begin{array}{c}2.375 \text { dia } \\
\text { x } 6\end{array}$ & 0.3 & 1000 & $\begin{array}{c}1.5 \mathrm{~A} \\
\text { start, } \\
800 \mathrm{ma}\end{array}$ \\
\hline $\begin{array}{l}\text { G-828 } \\
\text { (MFAM) }\end{array}$ & 2 & $\begin{array}{c}2.375 \text { dia } \\
\text { x 2.5 }\end{array}$ & 10 & 10 & $150 \mathrm{ma}$ \\
\hline & & & & & \\
\hline
\end{tabular}

Table 4.1. Characteristics of several Geometrics magnetometers. The G824 is the likely candidate for an airborne system

\subsection{Summary}

All critical components for a modular system are available at sufficient maturity levels. Thus, the conditions for the construction of a modular system are favorable. 


\section{Feasibility of modular approach: field testing results}

\subsection{Summary}

In order to asses both the feasibility of modular component integration into a system and the performance of different aspects of autonomous UAV rotorcraft several field testing efforts were undertaken. This chapter reports on these results. Two field campaigns were undertaken.

The first one (in May of 2009) assessed the feasibility of the following aspects

1. The ability of an autonomous UAV rotorcraft system to follow terrain

2. The ability to integrate a basic magnetometer on an autonomous UAV rotorcraft system and do real time data acquisition and telemetry

The second test (in November of 2009, conducted in Trenton, Florida) assessed the feasibility of the following aspects

1. The ability of the rotorcraft controller to be tuned such that it is able to deal with a boom structure on an autonomous UAV rotorcraft

2. The ability to collect high quality magnetic data from modular helimag system (flown manually)

Both of these tests were successful in their respective objectives. From this we conclude that both our original premise (that autonomous UAV rotorcraft magnetometer systems can be successfully constructed) and the premise investigated in this report (that a modular approach is likely to be the most successful one) are correct.

\subsection{Field test 1: Assessment of terrain following capability and component integration}

The objective of the first field test was to assess the feasibility of the following aspects

1. The ability of an autonomous UAV rotorcraft system to follow terrain

2. The ability to integrate a basic magnetometer on an autonomous UAV rotorcraft system and do real time data acquisition and telemetry

The ability to autonomously terrain follow is a critical one. It requires that the controller utilize input from a real time height sensor into the control of the rotorcraft in such a way that the system follow terrain in a way which maintains an as constant as possible height above ground. Note that this ability is related to but different from the ability to avoid obstacles. The latter ability was not investigated in this effort. 
For this effort INL subcontracted with Guided Systems. Two platforms were used in this effort. For the development of the terrain following, Guided Systems used a Mongoose (SICX) platform which is equipped with a Piccolo II autopilot. The Mongoose (SICX) is a gas powered rotorcraft with a payload capability of approximately $12 \mathrm{lbs}$. This was equipped with a $30 \mathrm{~Hz}$ laser altimeter, and Guided Systems modified the control laws on the autopilot to utilize the laser altimeter data for terrain following.

This system was used for a number of test flights, and the remainder of this section discusses the results of these flights. Note that no magnetometer boom was installed on the helicopter for the test results presented in this section. Figure 5.1 presents a representative flight test result from the flight testing of closed-loop above-ground -level (AGL) altitude tracking conducted on the SiCX-12 Mongoose unmanned helicopter.
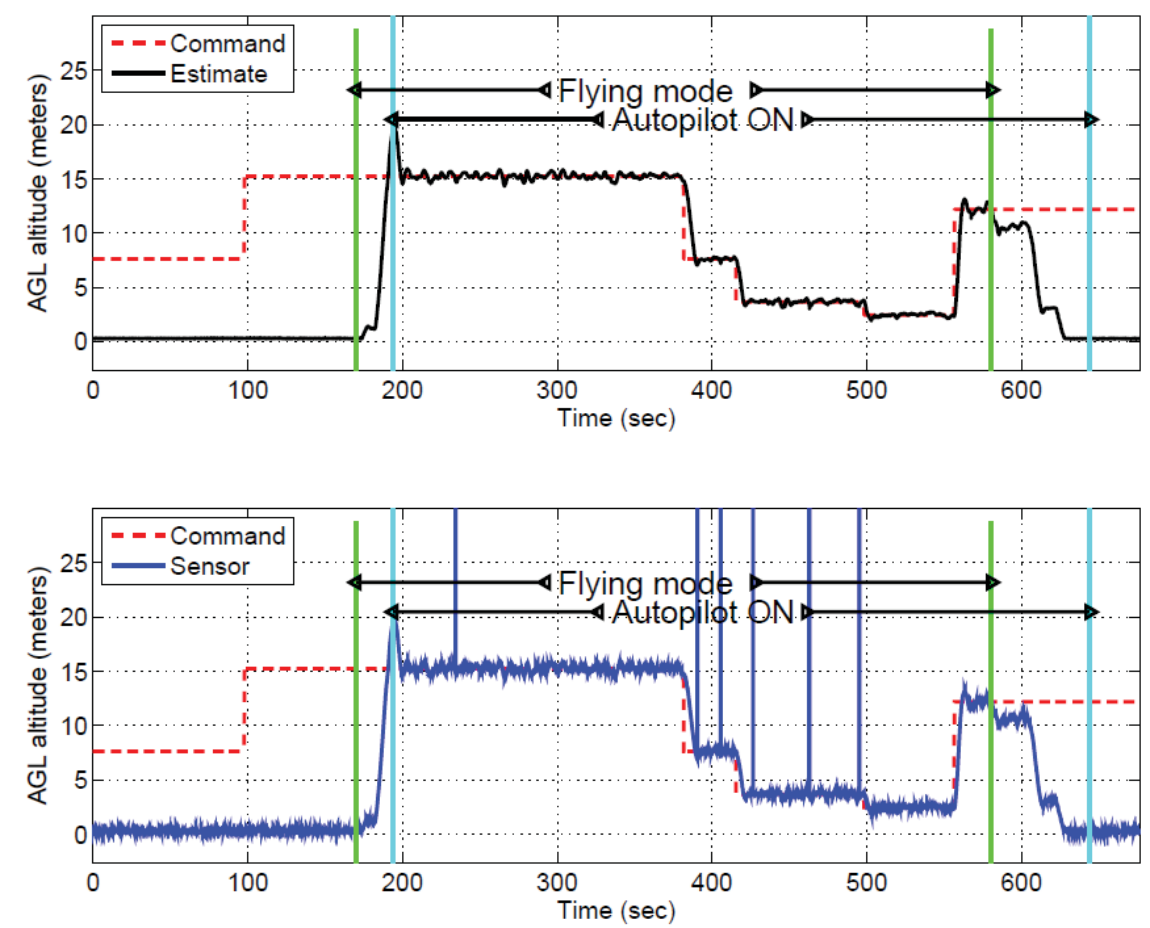

Figure 5.1 Initial flight test results of closed loop AGL tracking using Mongoose unmanned helicopter (filter output above, raw laser output below)

The upper half of Figure 5.1 presents the time history of both commanded (what the system is told to fly at) and measured AGL altitude for one of the flight tests. The measurement plotted is the estimate of AGL altitude produced by the navigation filter, which forms a best estimate using the the raw range data from the laser altimeter combined with inertial sensors and GPS. The entire flight operation from take-off (at about 180 seconds) to landing (at about 630 seconds) is shown. Note that the only portion of the flight where the AGL tracking mode is active and the measured vertical position of the helicopter should match the commanded height above ground is when the 
autopilot is on and in flying mode. Flying mode refers to a condition of the state machine implemented in software that manages automation of take-off, landing and flight between waypoints. Thus, as indicated by the labels in the figure, at about 180 seconds into the data record, the state machine entered flying mode even though the pilot had not yet turned the autopilot on (i.e. the conditions required to enter flying mode had been met). At this same time the pilot manually commanded climb of the helicopter to about 20 meters. Shortly thereafter the autopilot is turned on with the AGL tracking feature engaged and an AGL command of 15 meters. The helicopter tracks the AGL command well for the duration of the test record, including step changes in the AGL command at about 380, 420, 500 and 560 seconds. At about 580 seconds the vehicle is commanded to land and exits flying mode to enter an alternate mode of the state machine that handles the landing task and does not employ the same AGL command.

The lower portion of Figure 5.1 presents data for the same flight, again comparing the commanded height above ground and the response, but this time presents the raw height data being output by the laser altimeter in blue. Spurious data points are clearly evident in the time history, illustrating the importance of the navigation filter.

Figure 5.2 presents the time histories of the error between the AGL command and the AGL response (again using the filtered output above, and the raw laser output below).
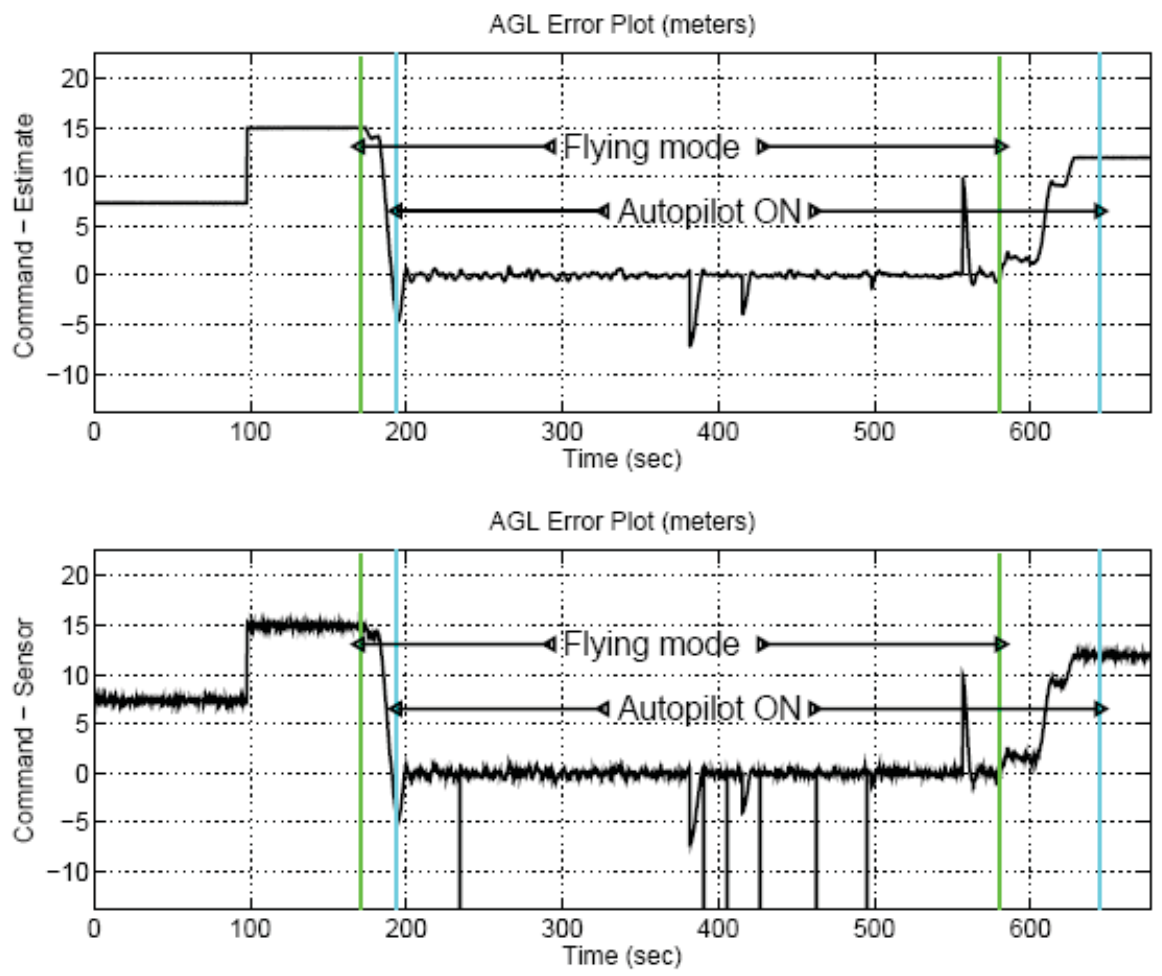

Figure 5.2 Time histories of errors between AGL command and response corresponding to flight test results of Figure 5.1 (filter output above, raw laser output below). Scale is in meters. 
It is of interest to characterize the performance of the AGL tracking loop by analyzing this error data. However, it is important to recognize that at this point in the program the goal was simply to develop and demonstrate the feasibility of an AGL tracking loop, with no specific performance requirement being given. At present, the laser altimeter output is combined with inertial sensor data and a single point GPS solution to build an estimate of AGL altitude. No attempt was made to optimize the filter or controller performance to meet a specific requirement, and no evaluation was made of the influence that a precision GPS solution would have on the AGL estimate. In fact, the filter parameters where chosen such that step changes in altitude, as might occur when passing over a man-made object purposely placed on the field as a target, are smoothed. The laser sensor is a single point unit fixed in the airframe (i.e it does not scan over an area and produce an average). The laser does not see through vegetation as a radar of correct frequency can. The laser thus instantaneously "sees" the vegetation on the ground at a single point and its output includes the noise created by flight over this vegetation in motion, since the vegetation is being disturbed by the rotor downwash. Furthermore, the performance of the vertical control loop was adjusted simply to give a reasonable result based on a qualitative assessment derived from direct observation of the vehicle in flight. A command filter is also used at the input to the vertical control loop that has the effect of smoothing the response to a step change in the measured altitude. There were insufficient program resources to do anything more than this in the current phase, and one must be careful to take these many factors into consideration when drawing conclusions from analysis of the data presented in this report.

With this caveat in mind, it remains of interest to characterize the performance of the AGL tracking loop by analyzing the error data presented in Figure 5.2. During the transient response to step changes in the commanded height above ground level, large error spikes occur (at about 380, 420, 500 and 560 seconds). It is anticipated that magnetometer data collection for survey purposes would only take place while operating at constant altitude, thus the errors in tracking AGL altitude associated with these transients should be excluded from the error analysis. One simple way to process the flight test data record without having to manually extract data records that exclude these transitions is to employ an error histogram.

Figure 5.3 presents such an error histogram generated from the data presented in Figure 5.2 when AGL tracking is active using error bins of width 0.25 meters. The total number of samples is 4800 . The number of samples with tracking error between -0.25 and +0.25 meters is 3499 . Thus the probability of an error between -0.25 and +0.25 for this data record is $3499 / 4800$ or $72.9 \%$. The probability of an error between -0.75 and +0.75 meters is $(528+3499+492) / 4800$ or $94.2 \%$. The standard deviation for the error plot of Figure 5.2, without excluding the large errors that occur during transitions in altitude is 1.0796 meteres. As this data was obtained without significant efforts to tune and refine the system performance we feel that this data evidences the ability to successfully follow terrain. 


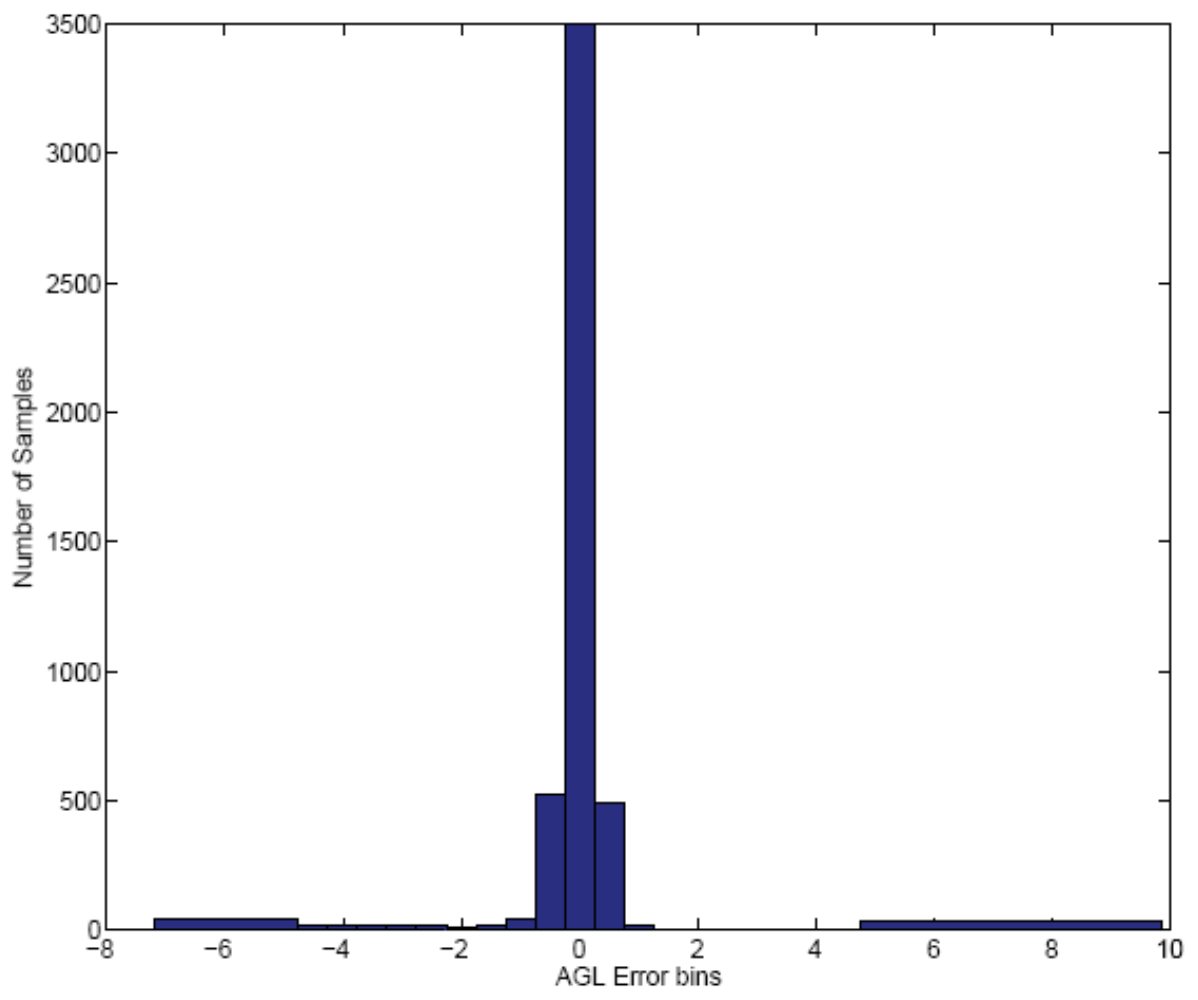

Figure 5.3 Error histogram for test data presented in Figure 5.2

Subsequent to the tests described above (which used the SICK-12 Mongoose) a test was performed which comined terrain following and real time magnetic data acquisition. For this a platform owned by INL (a Maxi Joker) was used. This platform is equipped with a Gimbal Pilot, which employs the same electronics and software as the Guided System Piccolo II autopilot. An Applied Physics 534D three component fluxgate magnetometer and a $30 \mathrm{~Hz}$ altimeter were also mounted on this platform. The data stream of the Applied Physics magnetometer was integrated into the Piccolo autopilot communications. This enabled us to stream sensor data directly to the ground station recorder during flight.

The capability for terrain following which was developed on the SICK-12 Mongoose was integrated (in about 4 days) on the Maxi Joker controller (demonstrating the portability of code as well as the advantages of modularity). A test flight was performed to demonstrate terrain following capability and data acquisition. The results are shown in figure 5.4 and 5.5. Figure 5.4 shows a composite picture of the results of two flights with terrain following turned on and off, while figure 5.5 only shows the terrain following flight. For the terrain following flight shown in figure 5.4 the controller was commanded to hold altitude starting at $8 \mathrm{ft}$ above the ground and fly from a GPS waypoint on the right to a GPS waypoint on the left of the image and back again. Subsequent to this, terrain 
following was turned off, and the same flight was repeated, Figure 5.4 and 5.5 illustrate visually the terrain following ability of the system shown in figures 5.1-5.3.

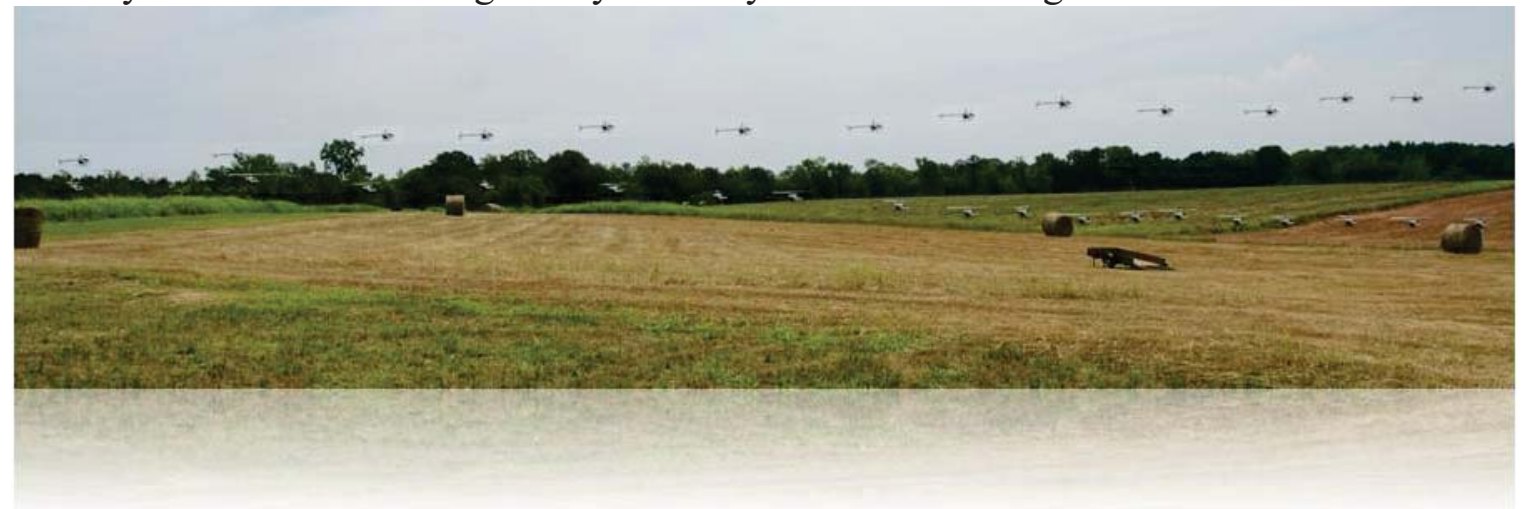

Figure 5.4 Results of terrain following test using the INL Maxi Joker comparing terrain following flight vs non terrain following flight.

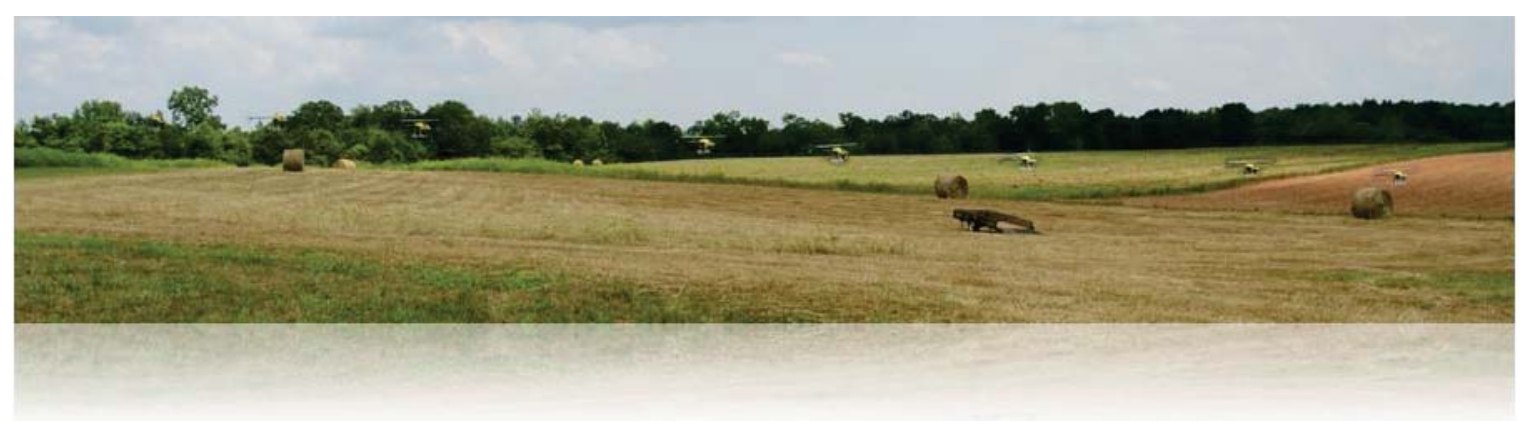

Figure 5.5 Composite image of the terrain following flight

As part of the terrain following tests with the MaxiJoker magnetic data was collected and streamed in real time to the UAV Operator Control Unit. To assess the performance of the magnetic data acquisition data was collected both at $8 \mathrm{ft}$ and $6 \mathrm{ft}$ height above ground. In order to obtain a clear magnetic signature data a trailer with some metal was placed underneath the flightpath (Figure 5.6)

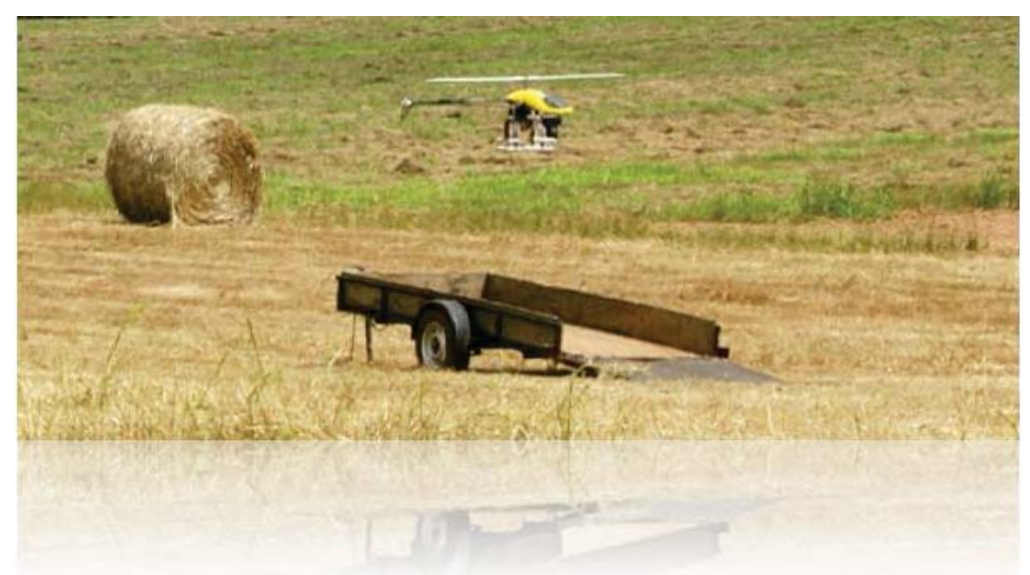

Figure 5.6 INL Joker passing over the trailer used as a target 
Figure 5.7 shows the results of test flights at $8 \mathrm{ft}$ and $6 \mathrm{ft}$ above ground. During these tests the helicopter flies over the trailer multiple times. The value of the total magnetic field is shown.

d

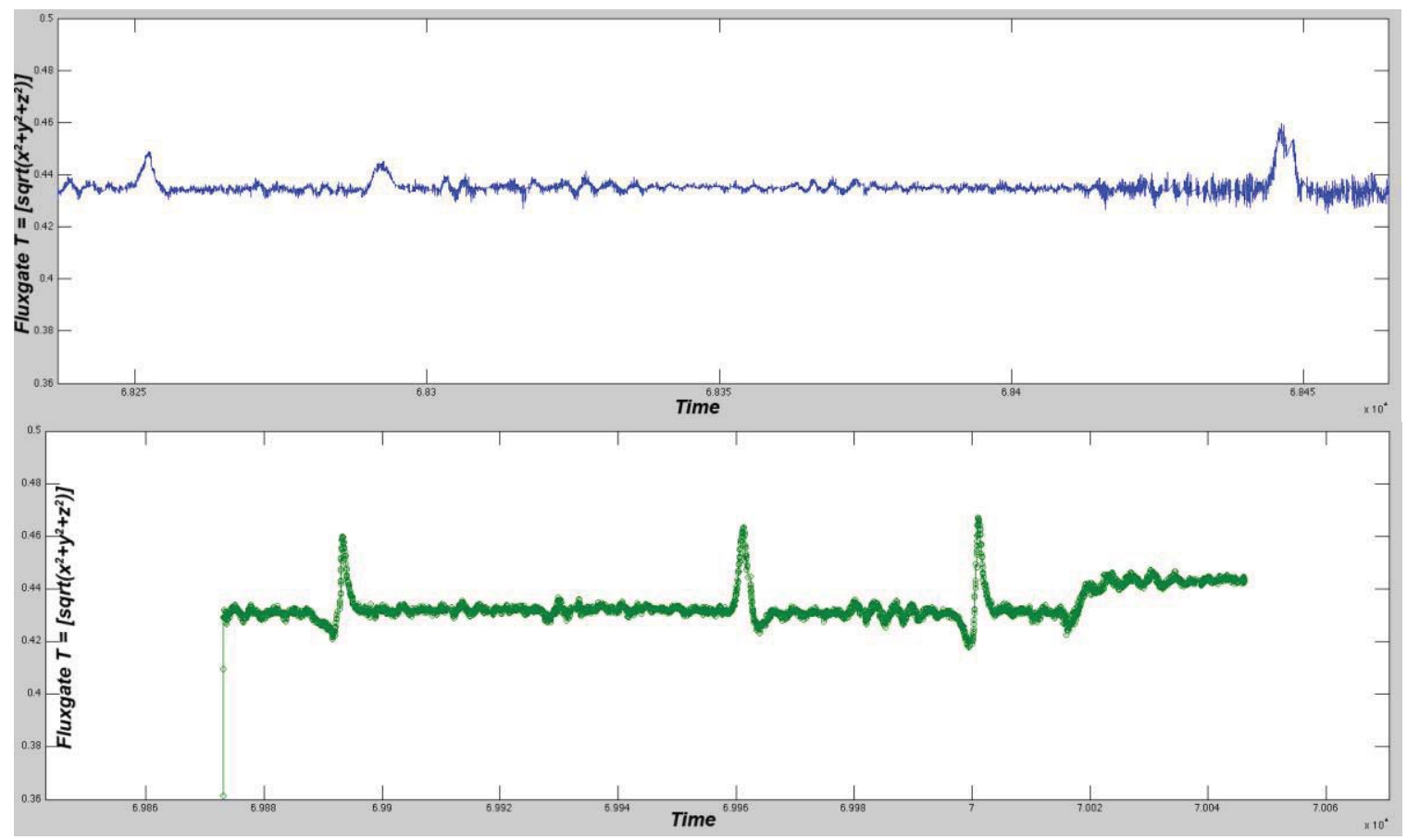

Figure 5.7 Value of magnetic field at different altitudes. Top: result of flight at $8 \mathrm{ft}$. above ground at 2 Knots. Data peaks correspond to the helicopter passing over the trailer. The speed was changed from 2 to 1 Knot for the second part of the first test. Bottom: results at $6 \mathrm{ft}$. above ground with a speed of 2 Knots.

\subsection{Field test 2: Assessment of ability to tune the controller to a boom and ability to collect high quality magnetic data from a modular helimag system}

The results of the first field test were very encouraging, and following these additional funding was obtained from the program office for a second field test. The objective of this was to assess the feasibility of the following aspects

1. The ability of the rotorcraft controller to be tuned such that it is able to deal with a boom structure on an autonomous UAV rotorcraft 
2. The ability to collect high quality magnetic data from modular helimag system (flown manually)

As this test consumed a substantial part of the projects resources extensive planning was undertaken to ensure that all data collection objectives would be met. This was done in discussion with the program office, which was provided with the test plan and which provided detailed input into the test plan.

Two parallel efforts were undertaken. In the first one, the Mongoose system owned by Guided Systems was equipped with a boom. The boom was designed by Geometrics and constructed by INL. The boom does not have any sensors integrated in it, but was meant to be a prototype of a boom which would be utilized on this class of rotorcraft. The autopilot on the Mongoose was then tuned for the boom, and test flights were conducted to assess the stability of the system with a boom as well as to collect more data on the ability to do terrain following.

In the second effort, a Mosquito (a single seater rotorcraft designed and constructed by Composite Systems FX in Trenton, Florida) was equipped with a two magnetometer boom designed and built by Geometrics., a logging system, differential GPS, laser altimeter and a fluxgate magnetometer. This system was then flown by a pilot over a number of targets provided by Battelle to assess the ability to collect high quality data with a modular system

\subsubsection{Mongoose test: assessment of ability to tune autopilot controller to boom}

In order to assess the ability of the autopilot to be tuned to fly a rotorcraft with a boom the Mongoose platform was equipped with a boom (Figure 5.8 and 5.9). The boom extends outside of the rotor blades. The distance between the extremity of the boom and tail rotor is about 9 feet.

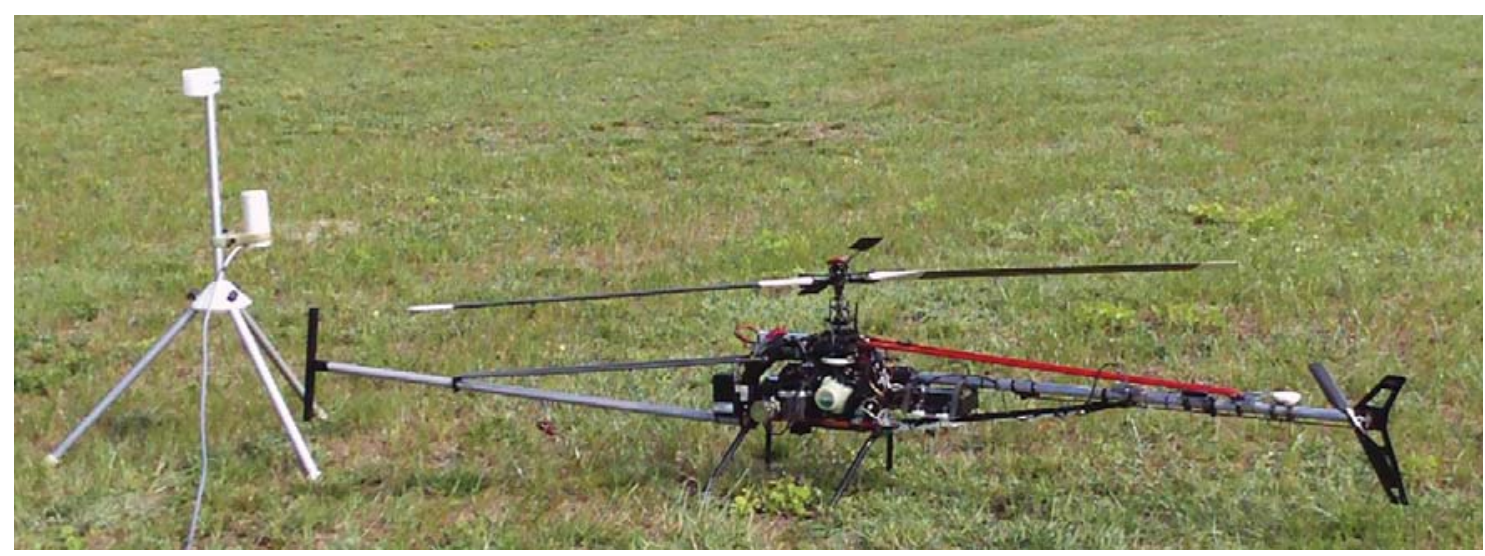

Figure 5.8 Mongoose with Boom 


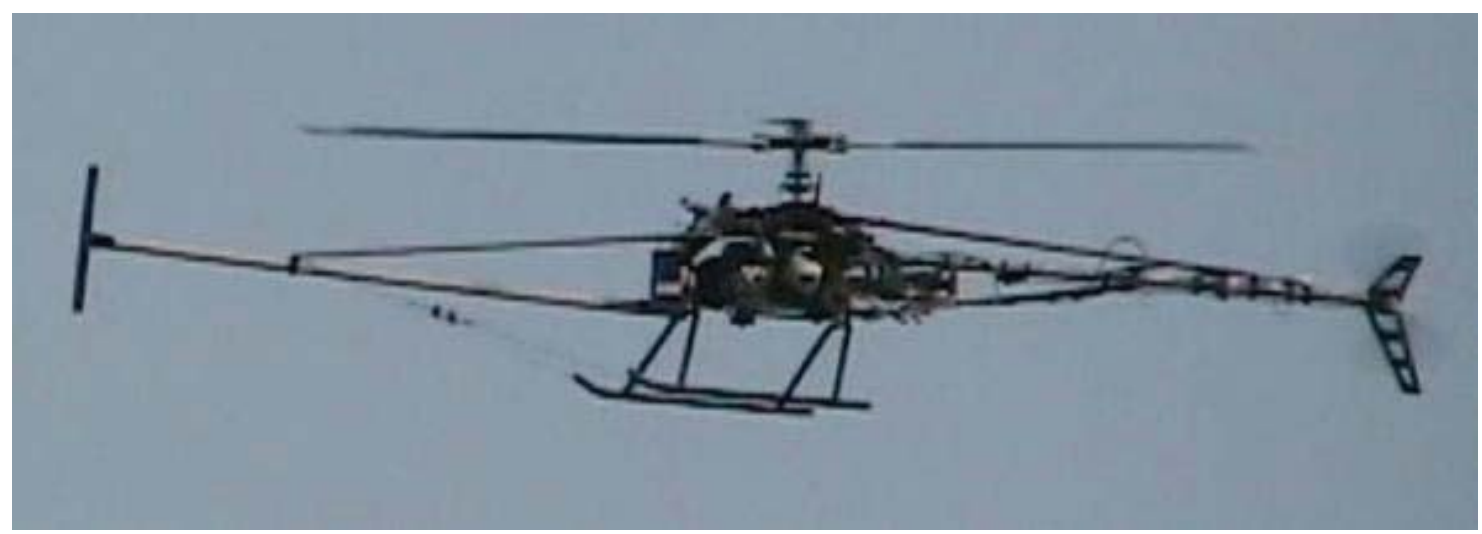

Figure 5.9 Mongoose with boom in flight

The addition of a boom to the helicopter will significantly alter the helicopter's mass properties, especially if the helicopter is relatively small. To appreciate the change in properties as well as the resulting impact on flight control it is necessary to understand the components of the system and their interplay.

The helicopter fundamentally uses tilt of the main rotor tip path plane relative to a body fixed coordinate system (such as might be defined by the axis of rotation of the main rotor drive shaft) to vector the thrust of the main rotor for control of vehicle inertial position and velocity. This thrust vector acts at the rotor hub to counteract the weight of the helicopter and enable flight. Ignoring many of the details (i.e. the aerodynamics of the body and that of body appendages, the tail rotor, and the mechanical complexities of the rotor hub) one can think of the body of the helicopter as simply suspended beneath the rotor hub and rotated at any instant to position the center of mass of the body (relative to the point of action of the thrust vector at the rotor hub) so that in trimmed flight all forces and moments acting on the vehicle sum to zero.

The Guided Systems developed control system allows for commanding the helicopter's position and velocity (the so called "outer" loops). The outputs of the outer loops are commands of the attitude angles of the fuselage (i.e. pitch, roll and yaw) that are then tracked by "inner" loops using direct measurements of the body attitude angles. The output signals of the inner loops generate inputs to the main rotor mechanics that ultimate result in tilting of the rotor tip path plane, and thus vectoring of the rotor thrust. In the end, the outer loops generate changes in desired pitch and roll angles of the fuselage in order to follow commands in position and velocity, and the inner loops generate inputs to the main and tail rotor mechanics to vector the thrust and vary the torque in order to track the commanded fuselage attitude angles.

If we introduce a boom that alters the mass moment of inertia of the unmanned helicopter body, we can expect this in turn to alter the response of the vehicle to the control system, and, in a worst case scenario, perhaps even render the control system incapable of achieving a given performance specification that had been achieved without the boom in place. 
In the current phase of the program, it was of interest to demonstrate the feasibility of automatic control of the unmanned helicopter with and without a representative boom, and to begin to develop an understanding of the impact the boom may have on performance of the automatic control system.

Figures 5.10 and 5.11 present time histories of the tracking of pitch and roll commands during nominal flight operations with and without the boom pictured in Figure 5.9 fitted on the SiCX-12 Mongoose helicopter. The helicopter is command to travel along straight line segments at constant AGL altitude at a speed of 1 meter per second, with vehicle heading commanded to remain aligned with the velocity vector.

Unfortunately, identical data records were not collected with and without the boom installed for long straight lengths without turns. The data without boom presented in Figures 5.10 and 5.11 is of a single straight line run. The data with boom presented in Figures 5.10 and 5.11 is of a flight of a number of straight line segments connected by turns. None the less, a general comparison of the two cases is instructive.

A plot of the corresponding heading command and response for the boom off and boom on cases is presented as Figure 5.12. Note that for the case of boom off, the heading command being tracked is zero, which is the same as 360 degrees. Thus the data trace jumps back and forth between zero and 360 degrees. Though not evident in the figure, tracking of heading measurement (a measurement which is not perfect) is very nearly of zero error in the case without the boom. In the case with the boom, oscillatory deviations of 4 to 5 degrees about the nominal heading command are evident from a detailed examination of the data, although not visible in the plot presented. Note that as the vehicle deviates from the commanded heading, the components of velocity in the body frame that correspond to an inertial velocity vector will vary, and thus the commands for pitch and roll angles required to regulated speed along the commanded path in the inertial frame will vary (due to the fact that pitch and roll and defined in the helicopter body axis system, whereas position and velocity tracking is performed in an inertial frame). Due to this coupling, if an oscillation in the yaw response occurs about the commanded heading, this oscillation will show up in the pitch and roll commands.

At the top of Figure 5.10 is a plot of the raw and filtered pitch command generated by the outer loops (they are essentially indistinguishable on this time scale), and the pitch attitude response of the vehicle to the controller, without the boom installed. Deviations from the observed mean of 2.5 degrees peak at -2.5 and +7.5 degrees. This data set was collected at a single value of vehicle heading, thus no deviation in performance that might occur due to response to a prevailing wind, or due to errors in the heading calculation that vary as a function of the heading, will be evident in the boom off case.

At the center of Figure 5.10 is a plot again of the raw and filtered pitch command generated by the outer loops (again they are essentially indistinguishable on this time scale), and the pitch attitude response of the vehicle to the controller, but this time with the boom installed (and including some turns that connect straight line path segments). Deviations from the observed mean of 2.5 degrees peak at -4 and +7.5 degrees. 
In comparing the upper (without boom) and middle plots (with boom) of Figure 5.10, it is immediately evident that while the controller in both cases is doing a reasonably good job of tracking the pitch attitude command, the system with the boom is noticeably more oscillatory than without the boom, at least during certain segments of the run. A plot of the velocity response is presented in the lower portion of Figure 5.10. This is a plot of the longitudinal component of the horizontal velocity achieved as compared to the commanded value of 1 meter per second. Oscillations in the velocity response are clearly greater in the case of the boom.

Note that a 3 axis magnetometer array is routinely employed on the helicopter to determine magnetic heading, which is then corrected to absolute heading using a local correction factor. The process of determining heading from this array is dependent upon estimates of pitch and roll angles. It should be further noted that past experience has shown that slight misalignment of this magnetometer array relative to the helicopter body axis system, and/or bias of the sensor, can lead to minor oscillations in the tracking of inertial frame position and velocity (since the transformation of position and velocity errors in the inertial frame to the controls that are applied in the helicopter body frame depend on knowledge of the heading angle).

Note further that the introduction of a boom on the helicopter will dramatically alter the mass moment of inertia about the yaw axis, and that because of the long moment arm created by the boom, atmospheric drag acting on the elements placed at the end of the boom and forward of the helicopter can create a destabilizing disturbance to the vehicle in the yaw channel. Winds on the test date were noted at 10 to 15 knots (5 to 8 meters per second). The regulated speed of travel for the data presented was only 1 meter per second. Thus the wind was a major disturbing factor in the tests, especially in those cases where the vehicle was being flown around a square pattern. For a vehicle as small as the SiCX-12 Mongoose, it is anticipated that this is a significant factor in overall control system performance. However, it can likely be counteracted in practice simply by adding a much larger vertical tail to the aircraft.

The lower portion of Figures 5.10, 11 and 12 are all identical except for the choice of colors in the associated legend.

Examination of the segments of the center and lower portions of Figure 5.10 with the center portion of Figure 5.12, one finds that during flight on a heading of 180 (traveling south), the oscillations in pitch attitude tracking when the boom is present are much more pronounced. These oscillations are also mildly evident in the center portion of Figure 5.11, which presents the roll response with the boom attached. The fact that the oscillations is evident in both the pitch and roll axes (i.e. in both Figures 5.10 and 5.11) indicates that the primary reason the response differs from boom off to boom on is due to the outer loop tracking of velocity and in the tracking of commanded heading. In other words, the pitch and roll loops are tracking their respective commands reasonably well both in the case of boom on and boom off. It is variation in tracking of the velocity 
commands, and most importantly in tracking of the commanded heading, that leads to the differences in the two cases. The variation in performance is clearly heading dependent.
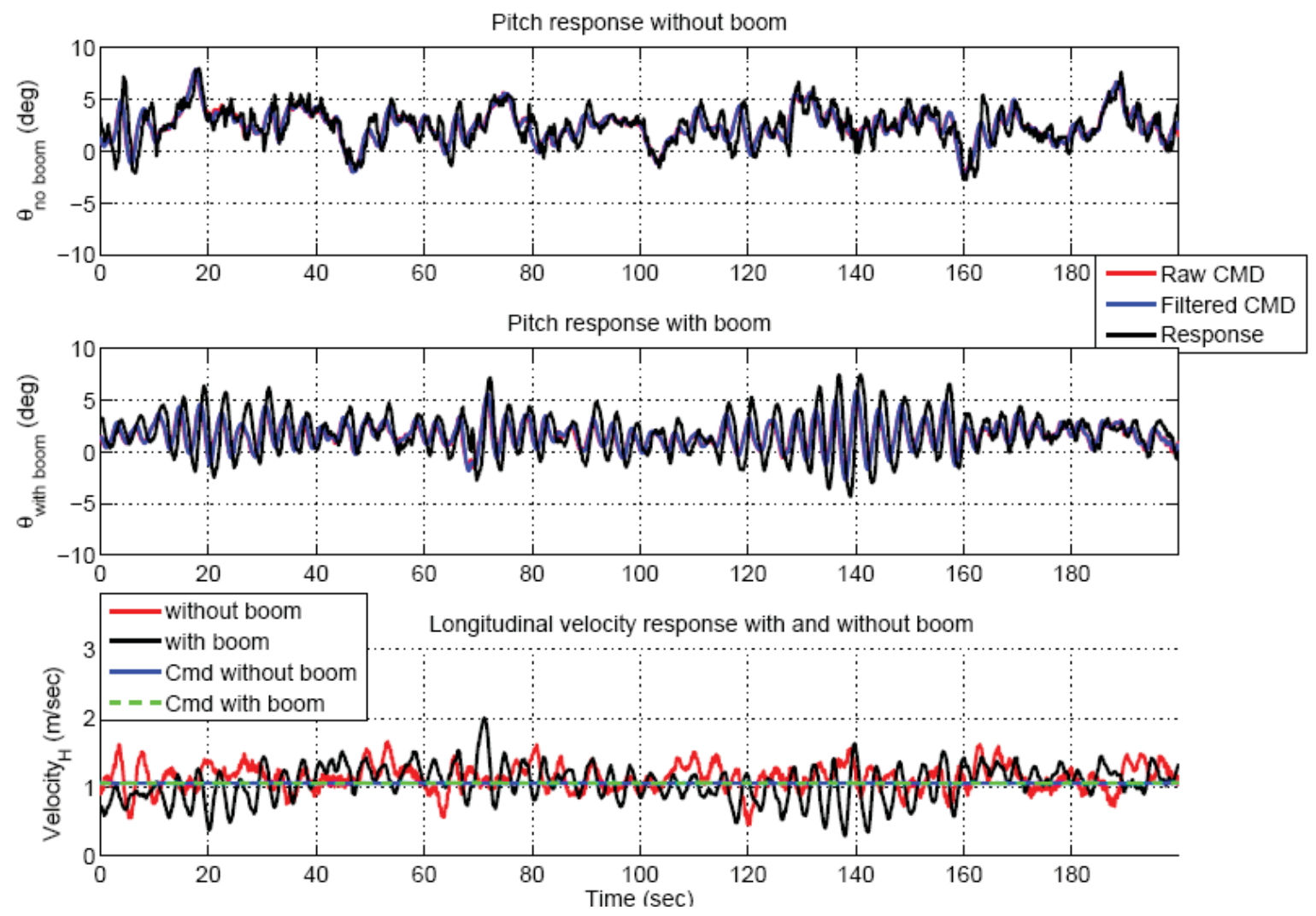

Figure 5.10. Time histories of pitch attitude tracking response on SiCX-12 helicopter with and without boom, and corresponding tracking of longitudinal component of horizontal velocity. 


\section{Roll response without boom}
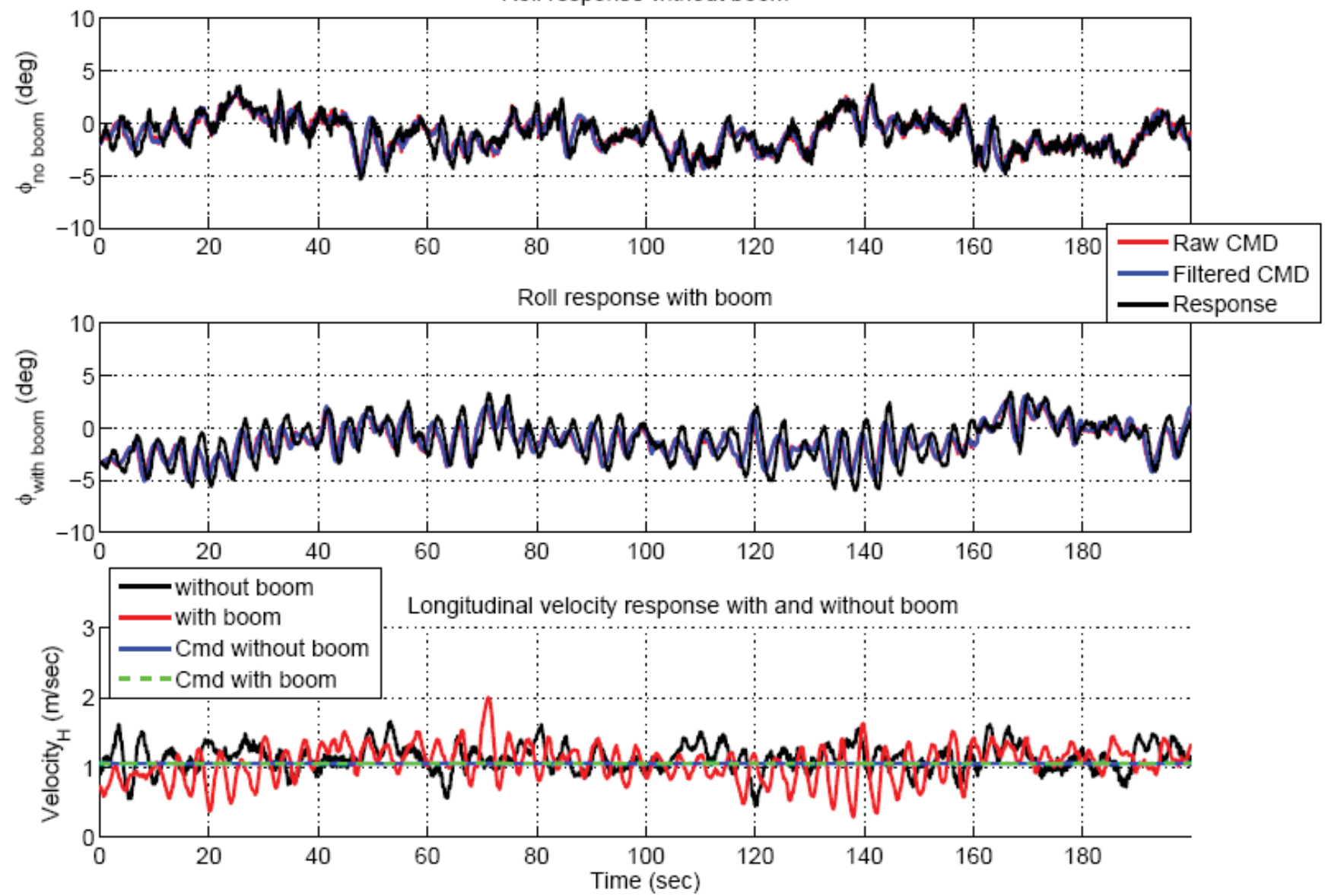

Figure 5.11. Time histories of roll attitude tracking response on SiCX-12 helicopter with and without boom, and corresponding tracking of longitudinal component of horizontal velocity. Case shown is the same as the case presented in Figure 5.10. 

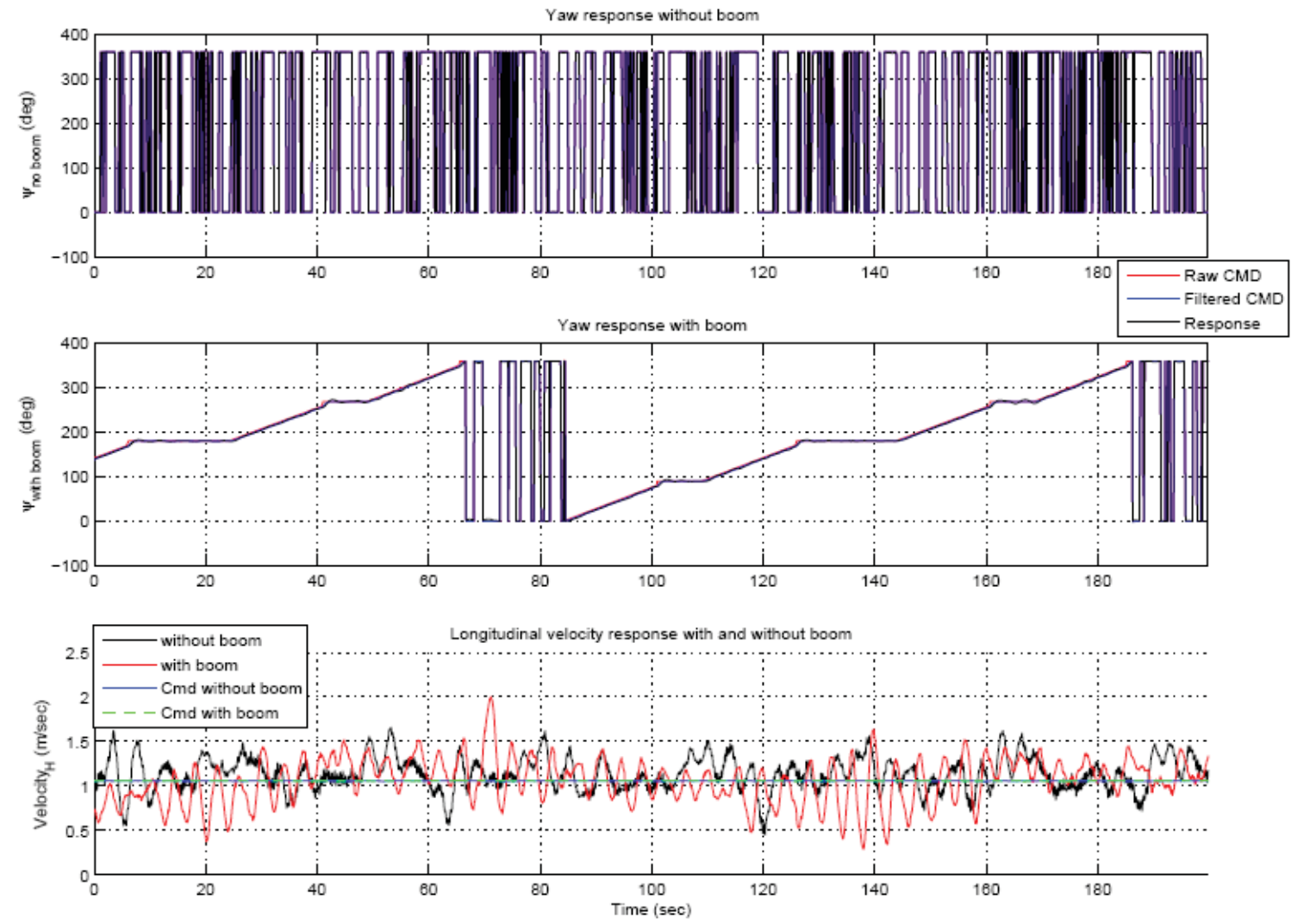

Figure 5.12 Time histories of yaw attitude tracking response on SiCX-12 helicopter with and without boom, and corresponding tracking of longitudinal component of horizontal velocity. Case shown is the same as the case presented in Figures 5.10 and 5.11 .

\subsubsection{Waypoint flights}

The data discussed in section 5.3.1 (which were collected by Guided Systems prior to the Trenton field effort) provided information on the stability of the Mongoose with and without a boom. During the Trenton field effort additional data was collected to further investigate the AGL altitude tracking performance of the SiCX-12 Mongoose helicopter with boom attached. For this test a simple square flight pattern was defined by a series of four GPS waypoints. The terrain of the test area consisted of a sloping hillside where the slope dropped to the east as well as to the south. The terrain altitude difference from the top left corner of the square to the bottom right corner was approximately 20 feet 
(roughly 6 meters). The square pattern was flown with a commanded AGL altitude of 10,8 and 6 feet for several different velocities.

Figures 5.13 and 5.14 present time histories tracking of AGL altitude commands (SiCX12 helicopter with boom attached) while flying the square waypoint pattern at a commanded AGL altitude of 10,8 and finally 6 feet. Note that figure 5.13 and 5.14 show the results of two different flights.
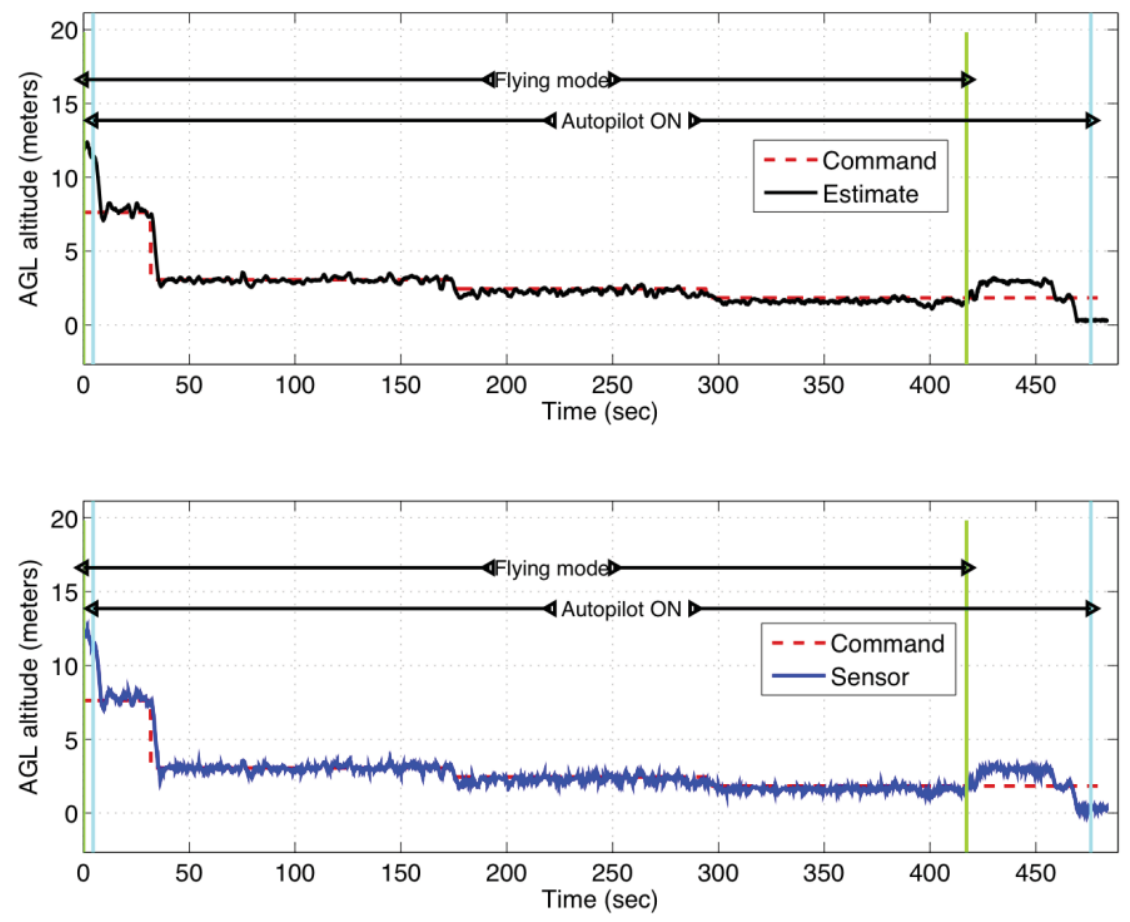

Figure 5.13. Data from terrain following for flight at 10, 8 and $6 \mathrm{ft}$. Top: filtered output, bottom: raw sensor output 

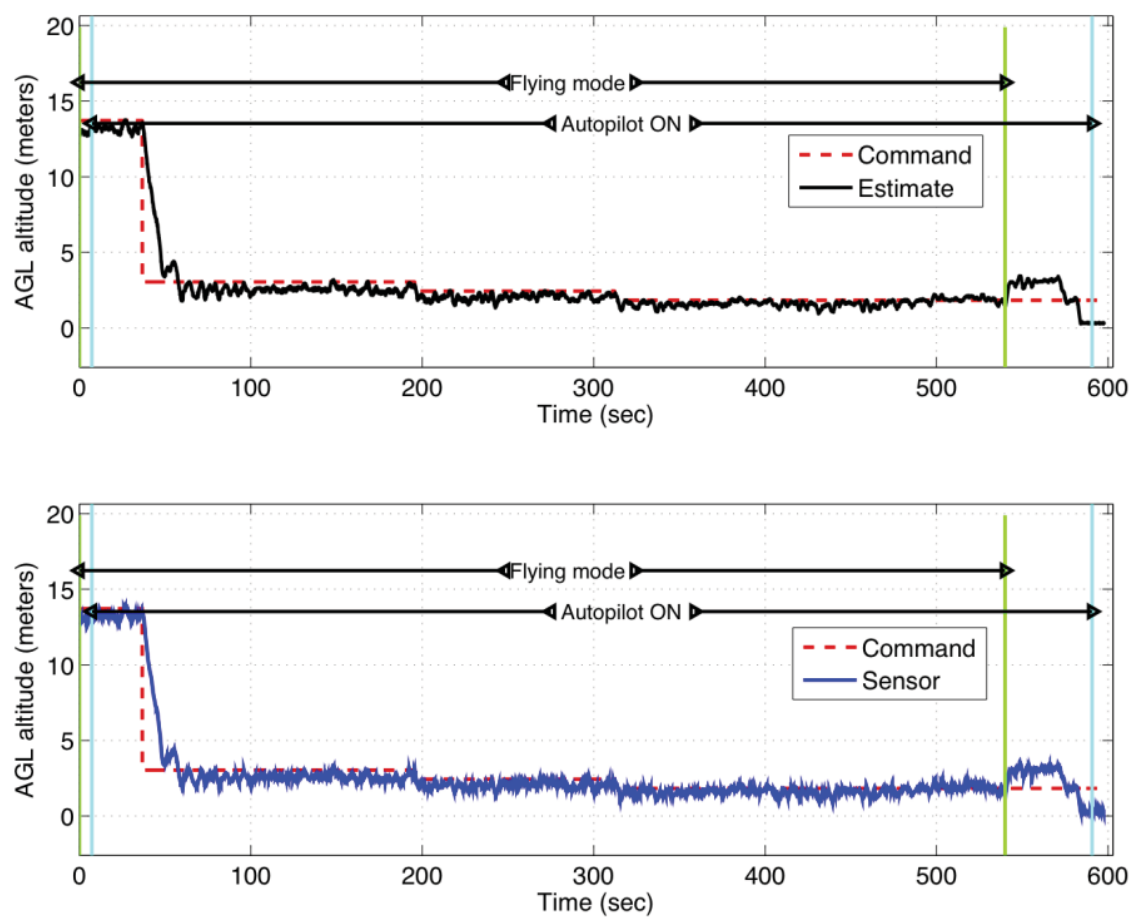

Figure 5.14. Data from terrain following for flight at 10, 8 and $6 \mathrm{ft}$. Top: filtered output, bottom: raw sensor output

As discussed in detail in Section 5.2, the only time AGL altitude tracking is active and AGL altitude is expected to match the AGL altitude command is when both the helicopter is in flying mode and the autopilot is on. As in Figure 5.2, the upper portion of figures 5.13 and 5.14 shows the AGL estimate generated by a filter and the lower portion of the figure presents the raw laser AGL data.

Error histograms were prepared for that portion of the flight where AGL tracking is active. These are presented as figures 5.15 and 5.16, corresponding to the data shown in Figures 5.13 and 5.14, respectively. 


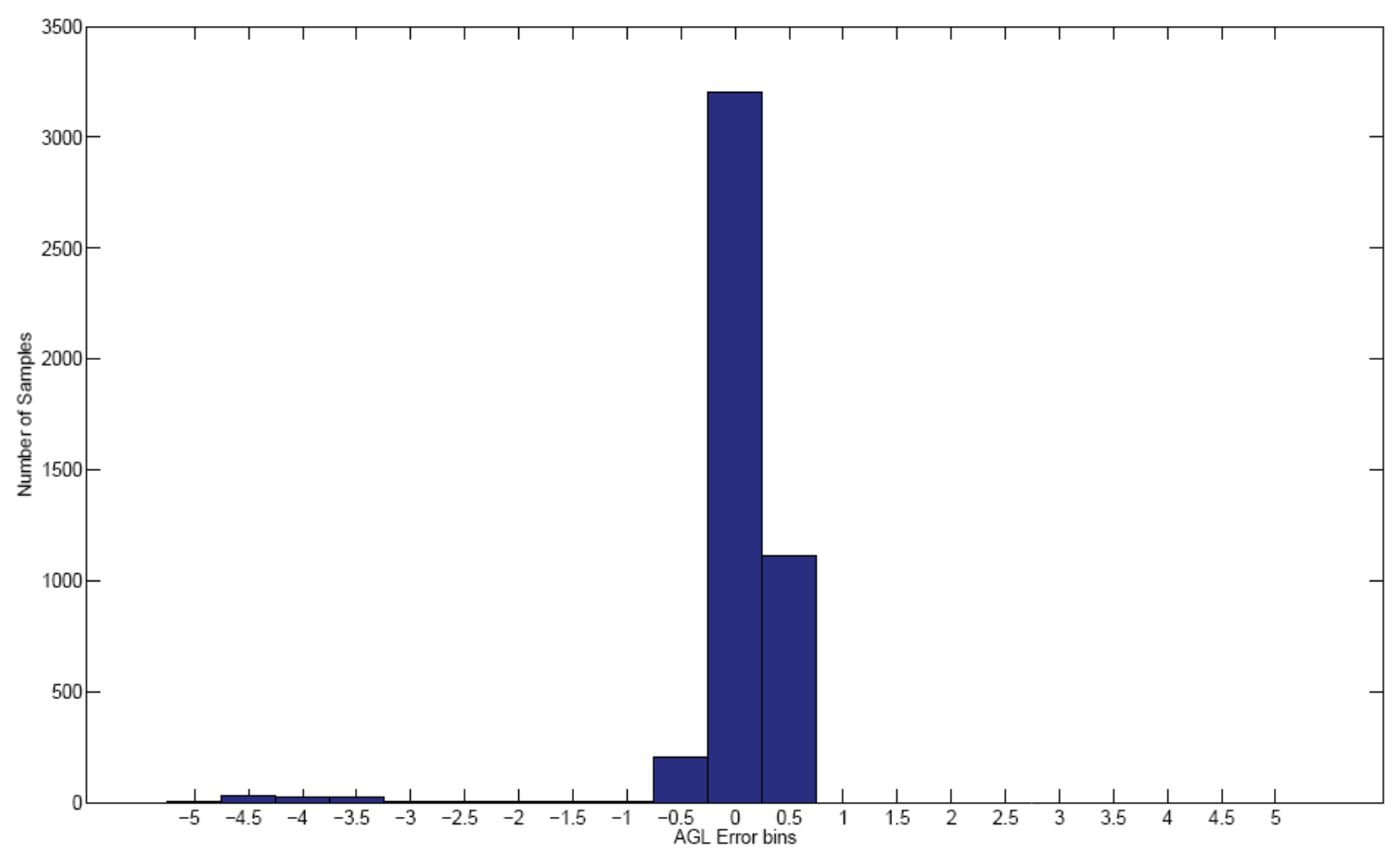

Figure 5.15 Error histogram for test data presented in Figure 5.13

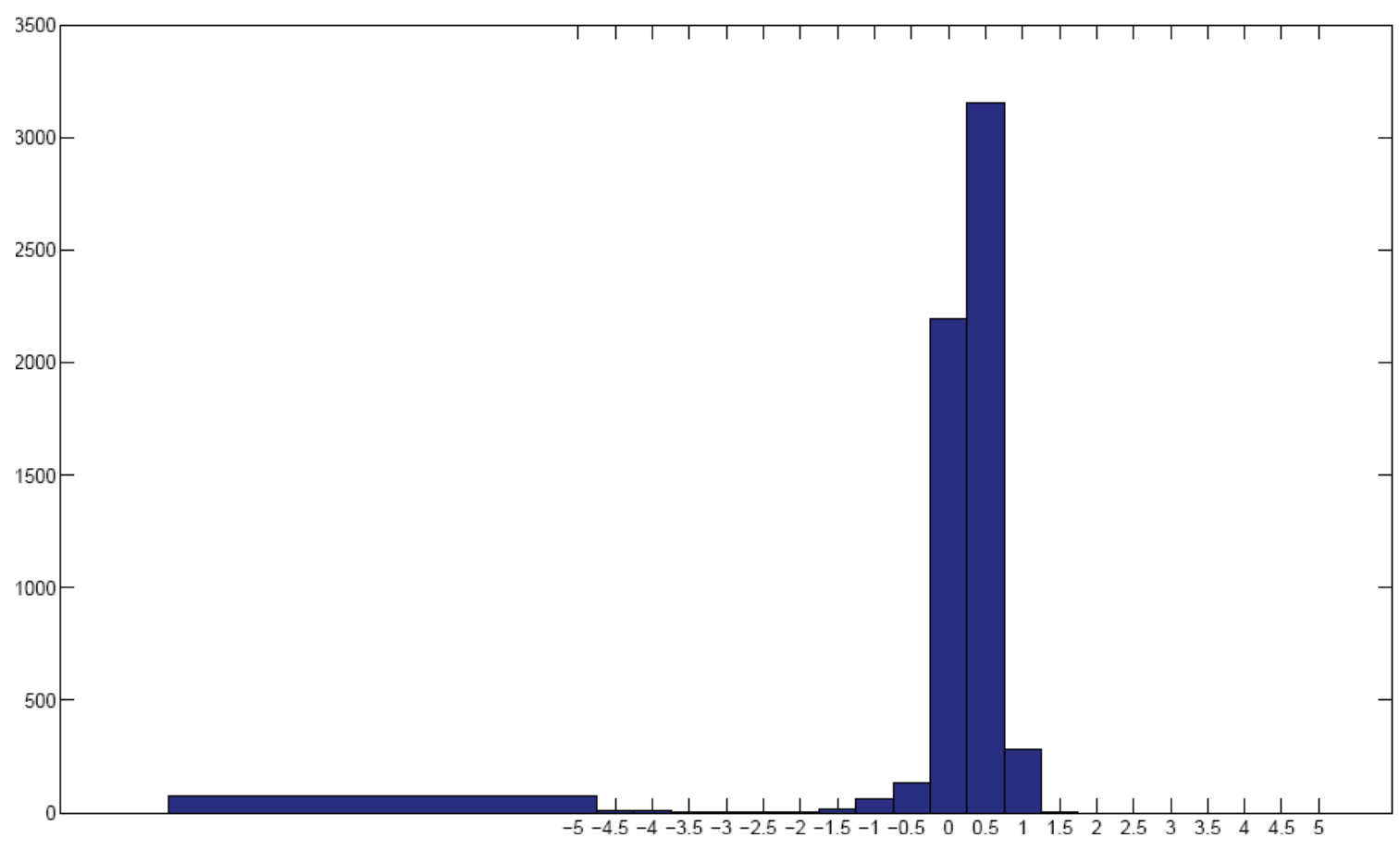

Figure 5.16 Error histogram for test data presented in Figure 5.14 
Table 5.1 summarizes the results presented in section 5.2 and here. Clearly the inclusion of the boom as well as turns has an impact, but the ability to maintain the AGL tracking errors to within + or -0.75 meters without any optimization of the sensor, filter or controller performance was only marginally effected by the presence of the boom.

\begin{tabular}{|l|l|l|l|}
\hline & Std Dev & $\begin{array}{l}\text { Altitude within } \\
\text { +/- 0.25 m of } \\
\text { commanded } \\
\text { altitude }\end{array}$ & $\begin{array}{l}\text { Altitude within +/- } \\
0.75 \mathrm{~m} \text { of } \\
\text { commanded altitude }\end{array}$ \\
\hline No boom, no turns & $0.65 \mathrm{~m}$ & 69 & 97 \\
\hline Boom and turns (test 1) & $1.01 \mathrm{~m}$ & 53 & 94 \\
\hline Boom and turns (test 2) & $1.08 \mathrm{~m}$ & 73 & 94 \\
\hline
\end{tabular}

Table 5.1 Summary of AGL Tracking Performance with and without boom for SiCX-12 Mongoose

\subsubsection{Summary discussion on autopilot controller of system with boom}

Helicopter dynamics are well known to be complex and to involve a great deal of cross coupling between the various control channels. Minor oscillations about the commanded heading can lead to oscillatory commands for pitch and roll in order to maintain track along a straight line segment defined in an inertial coordinate system. The addition of a boom structure can significantly alter the vehicle's mass properties, as well as its aerodynamics in ways that result in significant challenges for the flight control system. Response of the flight system can vary with wind direction, as well as produce variation in performance with heading due to sensor misalignment.

The results presented here show that the pre-existing commercial off-the-shelf automatic flight control system for helicopters in combination with the Guided System control laws can be employed to stabilize and control a helicopter with and without a boom in place and to maintain commanded AGL within a reasonable range. The results also show that adding a boom impacts the precision of the system to terrain follow

These results were obtained without any attempts to optimize the performance of the control system. It is anticipated from past experience that the tracking performance of AGL can be substantially improved with improved sensors (and by possibly utilizing the sensors used for magnetic data acquisition to provide additional information on platform orientation) and improvements in filter and controller design. This would be part of a future effort. 


\subsubsection{Mosquito test: magnetic data collection using a modular helimag system}

The tests discussed in the beginning of this chapter focus on the autonomous rotorcraft component of the system. While magnetic data was collected on one of the tests using a fluxgate magnetometer a decision was made to also investigate the feasibility of collecting magnetic data with a modular helimag system. For this, a Mosquito (Figure 5.17, a single seater rotorcraft designed and constructed by Composite Systems FX in Trenton, Florida) was equipped with a two magnetometer boom designed and built by Geometrics., a logging system, differential GPS, laser altimeter and a fluxgate magnetometer. This system was then flown by a pilot over a number of standard targets provided by Battelle to assess the ability to collect high quality data with a modular system

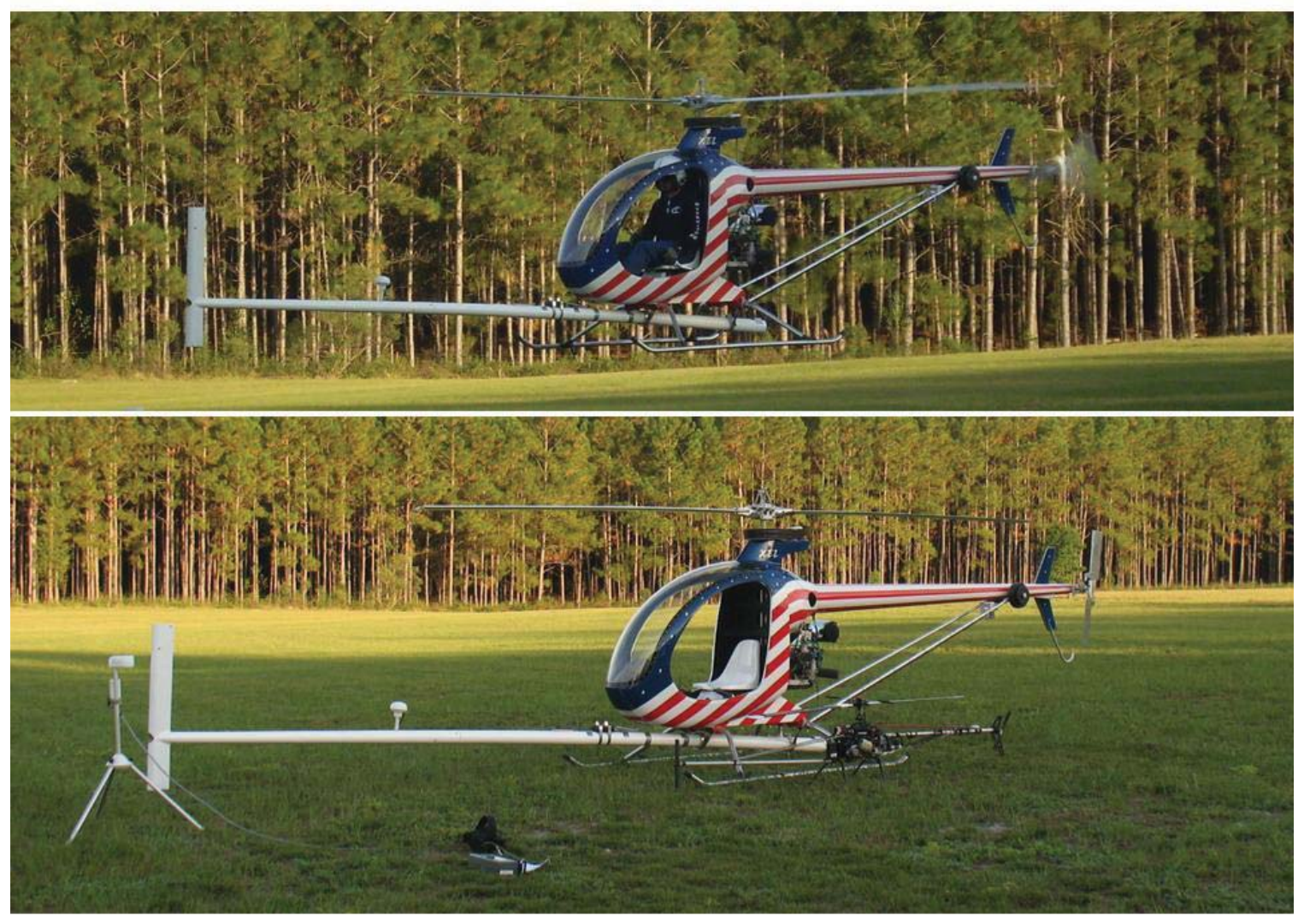

Figure 5.17 The Mosquito equipped with sensor boom. The system also has a DGPS unit, a laser altimeter and a fluxgate magnetometer on board. Data acquisition is done by a windows PC located in the cockpit adjacent to the pilot seat. All sensors are powered from $12 \mathrm{~V}$ batteries located in the cockpit.

\subsubsection{Boom description}


The prototype geophysical survey boom that was used in this effort consisted of a fiberglass pole with a gel coat finish (similar to the construction method employed in the Mosquito airframe). The pole was tapered from 4.25 inches to 3.0 inches to minimize moment-arm loading of the airframe. The cross tube on the forward end of the boom was designed to separate the two NIL G-823A sensors by one meter. The cross tube was made to accommodate the diameter of the sensors with a trailing edge fairing to minimize buffeting in forward flight. The materials chosen were wet-layup fiberglass cloth and vinyl-ester resin. A counterbalance approach was developed in collaboration between Geometrics and Composite FX to bring the aircraft back to its operating weight-andbalance envelope. For this, a seventeen pound counterweight was mounted on the helicopter's tail boom

The system flew well up to the designer-imposed VNE (Velocity to Never Exceed) of 40 Knots indicated airspeed. The biggest challenge with this type of boom design is to ensure that the boom's natural frequencies do not add with those of the aircraft (or vice versa) and create a vibration condition in the boom. This is important both for data quality and for safety consideration if vibration were to occur at higher speeds. The prototype boom developed for this project phase performed very well at all operating speeds.

\subsubsection{Data acquisition}

Data acquisition was done by Geometrics MagLog ${ }^{\mathrm{TM}}$ software which ran on a standard windows PC equipped with five serial ports. Data streams to these ports were as follows

1. Applied Physics 539 flux gate 3-axis magnetometer, running at approximately 15 $\mathrm{Hz}$ in ASCII with checksum mode, at 9600 baud.

2. Novatel Smart Antenna GPS running at $1 \mathrm{~Hz}, 9600$ baud and reporting GPGGA navigation messages. WAAS correction was partially available during the survey.

3. Opti-Logic Laser Rangefinder Module, running at 9600 baud, approximately $20 \mathrm{~Hz}$ sample rate, calibrated in feet.

4. Two "Geometrics" 823 magnetic sensors, concatenated together, with top sensor first, running at $10 \mathrm{~Hz}$ (some surveys at 20Hz), 9600 baud.

5. KVH C-100 magnetic compass running at $1 \mathrm{~Hz}$, outputting NMEA message, 4800 baud.

Pre-acquisition setup included the C-100 compass circular calibration. Unfortunately it turned out that the C-100 could not be calibrated correctly, and while data was collected during the field effort the compass readings are unreliable. Therefore the compass data are not used in the data analysis. 
The accuracy of the Opti-Logic Laser Rangefinder Module (altimeter) was tested prior to the flights. The accuracy of the values reported using this sensor during groundtests was low: with a sensor elevation of 3.5 feet above the ground readings of 2.5 and 6 feet were reported. Thus, while the in flight data indicate reasonable changes in elevation during the flight this data was not used in the analysis of the data presented below

In summary, the only data sets used for processing were GPS, Cesium magnetometers and flux gate magnetometer data.

As mentioned above, all data streams are recorded by MagLog ${ }^{\mathrm{TM}}$ software. The basic feature of this program is that raw data strings are concatenated with a PC timestamp as soon as they arrive at the serial bus. Following this, they are recorded to a flat file. Consequently, raw recorded data includes one file per device. The time stamps allow easy post processing and data stream correlation. In addition to the raw files one real-time interpolator file is created and recorded. This file contains GPS positions and data for the magnetometer sensor. These are calculated in real time during data acquisition from the GPS and magnetometer data streams.

As the main purpose of this test was to evaluate detectability of targets and not their precise positions no specific effort was made to identify and minimize latency issues Latency issues arise from a combination of issues: for instance, a device can delay transmitting data (for instance, NOVATEL GPS receiver sends GGA string 0.5 seconds after position fix). Another cause are delays in the serial reception on side of the host computer (typical values are 10-20 msec, depending on computer load). There are well known special ways to resolve these issues like using PPS (pulse per second) GPS output and special serial hardware and drives. However, as data acquisition rates were relatively slow, and as the flying speed was relatively low, it was felt that it was not necessary to devote project resources to latency minimization. Note that for a follow-up effort these latency issues would need to be identified and resolved.

\subsubsection{Survey site setup and magnetic data acquisition}

In order to assess the performance of the system an ad hoc test site was constructed at a field which is part of the Composite FX facility in Trenton. The selected area for the test site was surveyed for any magnetic anomalies prior to test site construction, and a number of small anomalies were identified and dug up. Subsequent to this, five parallel lines were measured out and marked with agricultural paint. Along the center line thirteen targets were laid out at $15 \mathrm{~m}$ spacing. The first target was at $0 \mathrm{~m}$, and the last target at 180 $\mathrm{m}$. Figure 5.18 and 5.19 show the targets used in the test.

Data was collected over this site both with and without the targets emplaced both with a handheld Geometric G823A gradiometer and with the Mosquito system. 


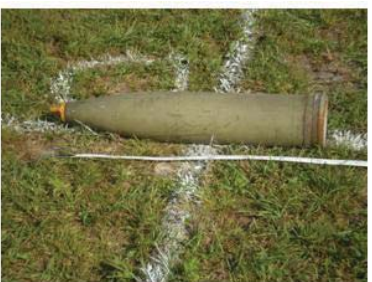

$155 \mathrm{~mm}$ artillery round $0 \mathrm{~m}$ position

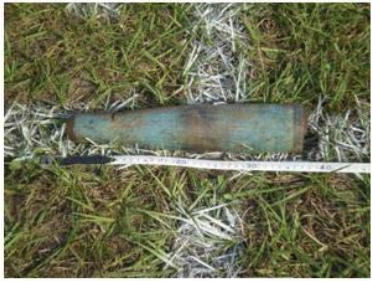

2.75 inch rocket warhead $45 \mathrm{~m}$ position

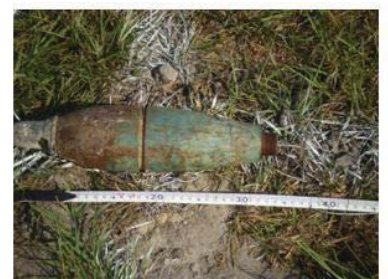

$81 \mathrm{~mm}$ Mortar Round $15 \mathrm{~m}$ position

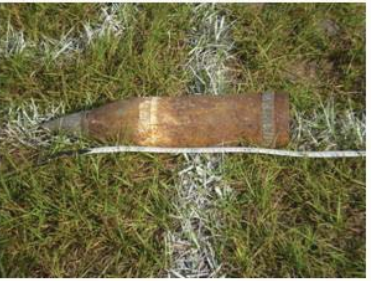

$105 \mathrm{~mm}$ artillery round $60 \mathrm{~m}$ position

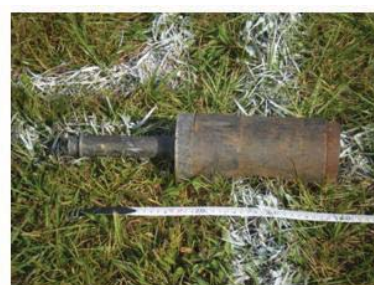

High Explosive Anti Tank (HEAT) Round, $105 \mathrm{~mm}$ $30 \mathrm{~m}$ position

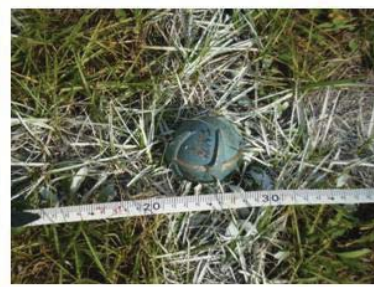

BLU 26 subminition $75 \mathrm{~m}$ and $105 \mathrm{~m}$ position

Figure 5.18 Targets used for magnetic detectability assessment survey.

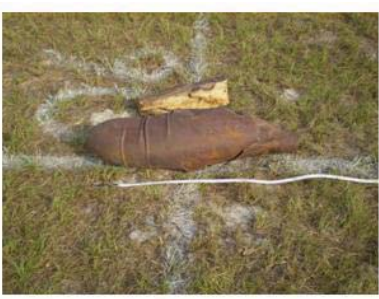

M-38 Practice Bomb Shell $90 \mathrm{~m}$ position

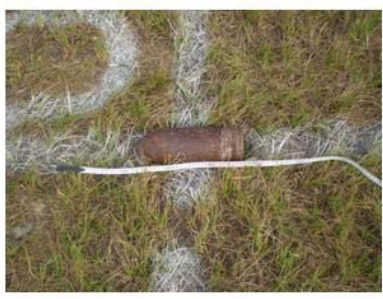

$90 \mathrm{~mm}$ artillery round $150 \mathrm{~m}$ position

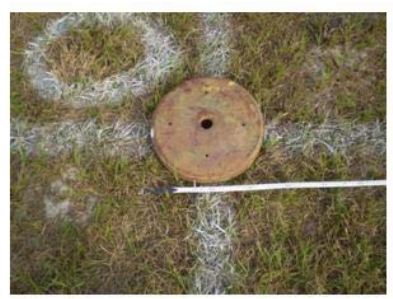

Anti Tank Mine $120 \mathrm{~m}$ position

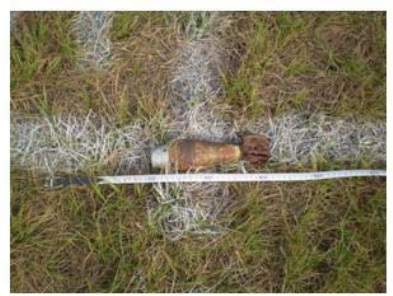

$60 \mathrm{~mm}$ mortar round $165 \mathrm{~m}$ position

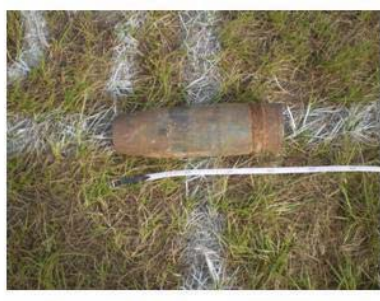

5 Inch Rocket Warhead $135 \mathrm{~m}$ position

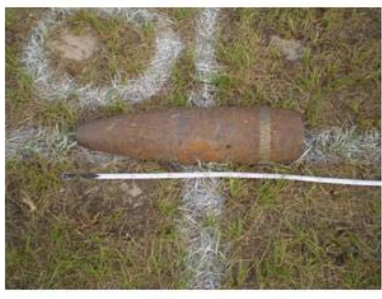

$155 \mathrm{~mm}$ artilerry round $180 \mathrm{~m}$ position

Figure 5.19. Targets used for magnetic detectability assessment survey. The targets at position $105 \mathrm{~m}$ are shown in figure 5.21 


\subsubsection{Data processing and results}

All data acquired during the surveys was re-positioned using the MagMap2000 GPS wizard feature. The procedure includes the following steps:

1. GPS positions were converted to UTM coordinates and linearly interpolated to the magnetometer time stamps. GPS PC time stamps were used. After this inverse UTM transformation was applied to express final positions in Longitude / Latitude. Elevation reported as part of GGA string was interpolated as well.

2. Flux gates readings were linearly interpolated to the magnetometer time stamps using PC time recorded with the flux gates.

3. Altimeter readings were linearly interpolated to the magnetometer time stamps using PC time recorded with altimeter data.

4. Magnetic Azimuth and Magnetic Roll angles were computed as Azimuth = atan2 $(Y, X)$ and Roll=atan2 $(Y, Z)$ Note that $X$ and $Y$ signs were changed for this computation because the flux gate magnetometer module was mounted backwards.

The same procedure was applied to the manually collected data. The results of both surveys are shown side by side in figure 5.20. Note that a DC shift was applied to the data Another way to look at this data is by comparing the data in profile format along the center line (Figure 5.21 and Figure 5.22) which shows the agreement between the two different datasets, or by comparing the amplitude and footprint of the targets between the land and the airborne survey (table 5.2).

The helimag system was able to detect all targets (and even allowed for the inversion for these data as shown in figure 5.20), and the data quality of the helimag system was comparable to that of the manual collected land data. As is expected from the difference in height in the survey altitude the footprint of the data for the airborne system is somewhat larger than the footprint of the data for the land dataset. Also, the min and max amplitudes associated with each target are lower for the airborne data than for the land data. 

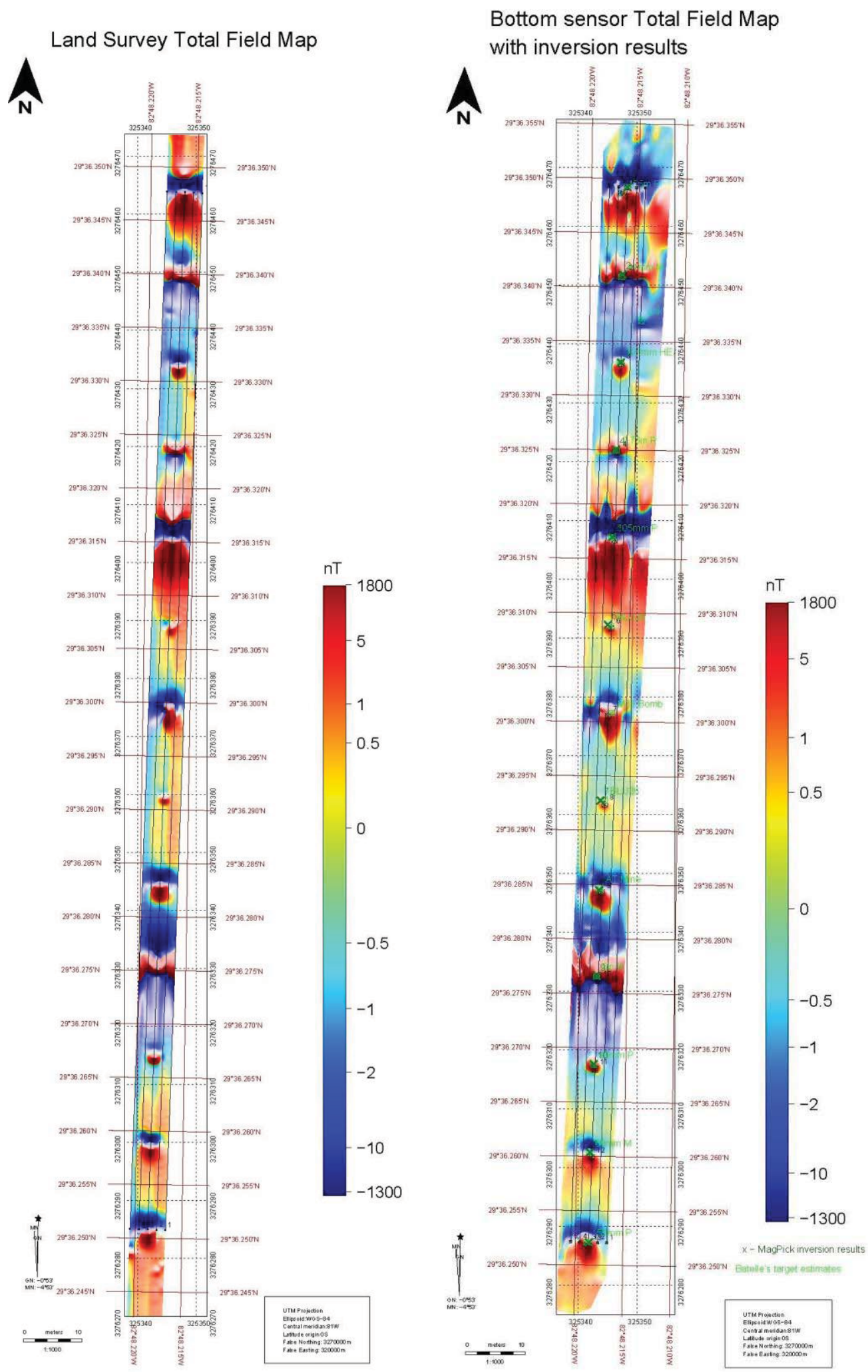

Figure 5.20. Manually collected data (left) vs data collected with helimag system (right) 


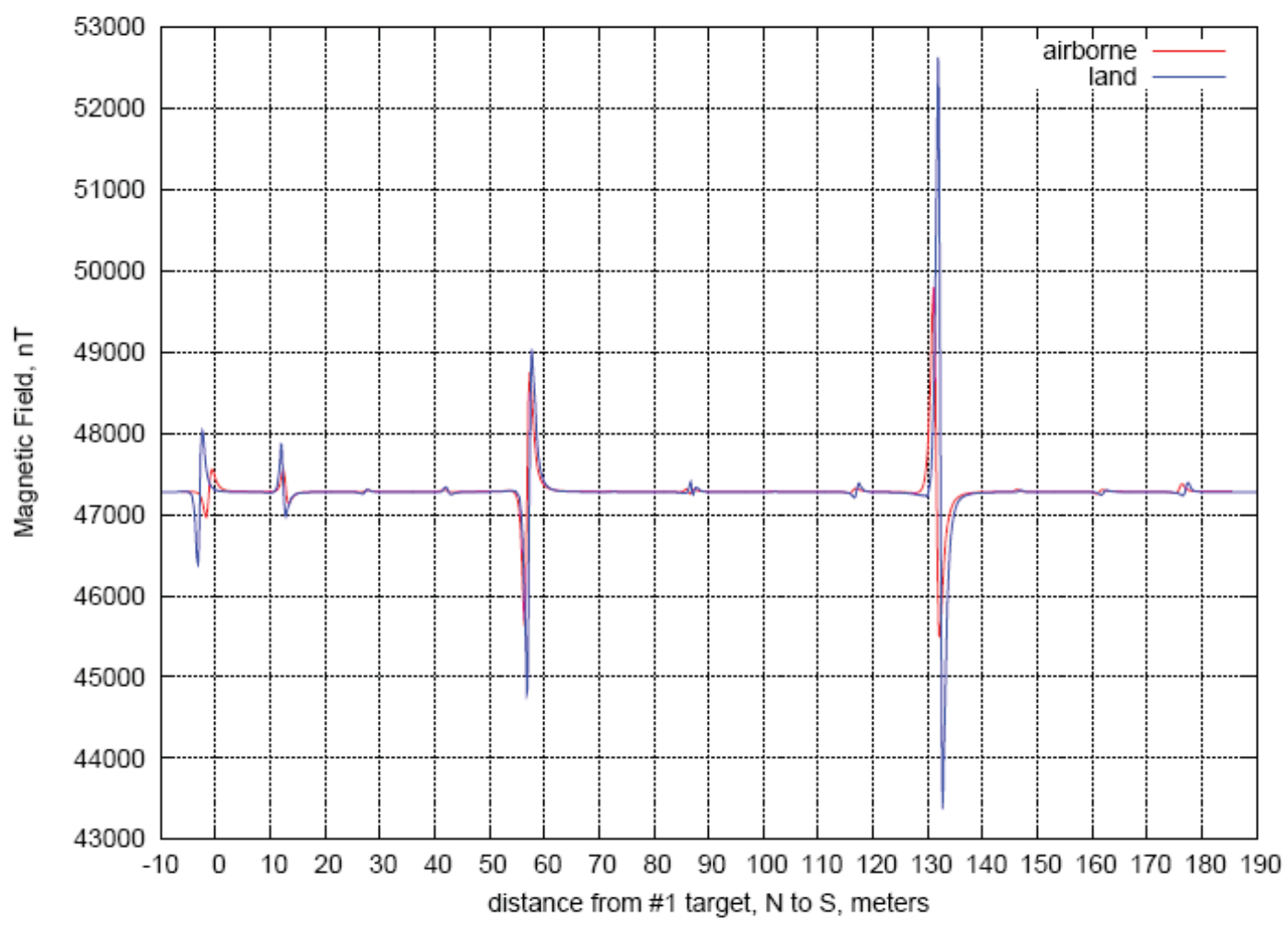

Figure 5.21. Comparison of the magnetic signatures of manually collected data vs airborne collected data over center profile line.. DC component shift has been removed from data. 


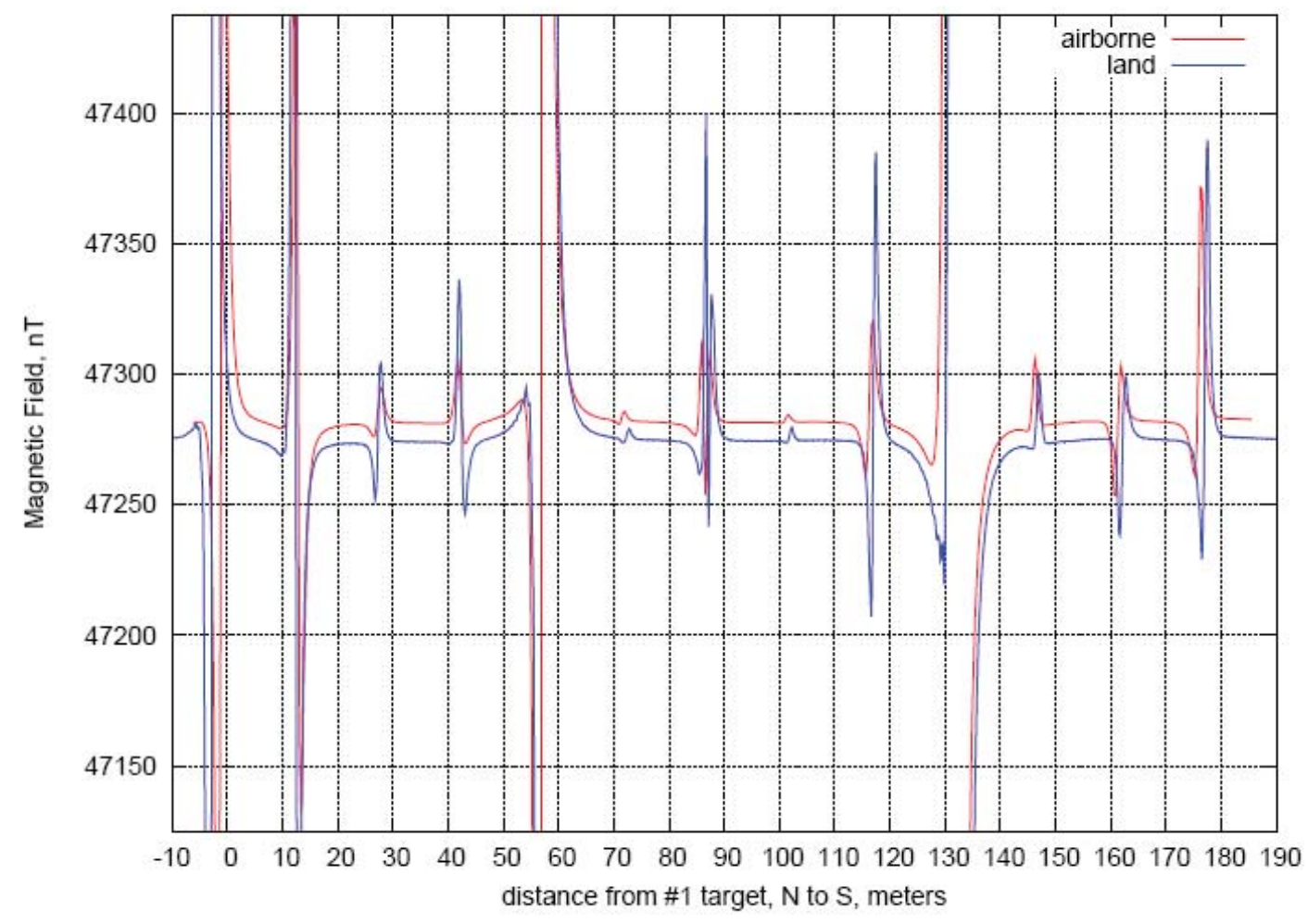

Figure 5.22. Same data as shown in figure $x \mathrm{x}$ with a narrower range showing correspondence of data

\begin{tabular}{|r|r|r|r|r|r|r|}
\hline Target_ID & Airborne Max & Airborne Min & $\begin{array}{l}\text { Airborne } \\
\text { footprint(m) }\end{array}$ & $\begin{array}{l}\text { Land } \\
\text { max }\end{array}$ & Land min & $\begin{array}{l}\text { Land } \\
\text { footprint(m) }\end{array}$ \\
\hline 1 & 82.25 & -85.93 & 2.36 & 652.13 & -692.14 & 1.00 \\
\hline 2 & 109.29 & -55.8 & 1.8 & 405.66 & -213.37 & 1.22 \\
\hline 3 & 9.15 & -3.99 & 1.9 & 24.61 & -16.63 & 1.26 \\
\hline 4 & 9.71 & -5.41 & 1.82 & 49.89 & -24.13 & 1.20 \\
\hline 5 & 592.92 & -728.16 & 1.6 & 1426.27 & -1932.6 & 1.10 \\
\hline 6 & 3.06 & 0.11 & 1.42 & 6.21 & -1.56 & 1.30 \\
\hline 7 & 46.15 & -6.3 & 2.44 & 184.66 & -151.95 & 1.12 \\
\hline 8 & 1.6 & -0.21 & 2 & 4.91 & -0.69 & 1.30 \\
\hline 9 & 53.04 & -19.76 & 1.5 & 93.89 & -45.98 & 1.10 \\
\hline 10 & 1870.67 & -1306.1 & 1.62 & 4090.17 & -3206.66 & 1.10 \\
\hline 11 & 10.95 & -2.75 & 2.68 & 22.16 & -3.1 & 1.60 \\
\hline 12 & 9.13 & -15.47 & 1.52 & 20.73 & -26.92 & 1.26 \\
\hline 13 & 39.73 & -9.63 & 2.12 & 115.3 & -41.28 & 1.28 \\
\hline
\end{tabular}

Table 5.2. Min and max of signal and signal footprint comparison for all targets 


\subsubsection{Aircraft signature measurements}

In addition to the site surveys reported on above the magnetic signatures of both the Mongoose and Mosquito rotorcraft were measured. In addition to the Mosquito used in the flight test an additional Mosquito with slightly different performance characteristics (Mosquito Turbo) was measured.

The signature measurements were carried out by approaching an 858 magnetometer sensor located in a fixed position from all four cardinal directions by moving the rotorcraft manually. This allowed for the estimation of possible induced and permanent components of the static aircraft magnetization as well as rotor effects. The Mongoose rotor was found to be highly magnetic and measurements for the Mongoose were repeated with the rotor removed, but the servo motors still running. Table 5.1 below summarizes measurements of three aircraft. These results indicate that the Mosquito is a magnetically very low noise aircraft, opposite to the Mongoose which exhibits values typical for helicopters. Note that apart from removing the rotor no effort was made to optimize the Mongoose with regard to magnetic signature.

\begin{tabular}{|l|l|l|l|l|l|l|}
\hline & Mongoose & $\begin{array}{l}\text { Mongoose } \\
\text { no rotor }\end{array}$ & $\begin{array}{l}\text { Mosquito } \\
\text { with boom }\end{array}$ & $\begin{array}{l}\text { Mosquito } \\
\text { Turbine } \\
\text { without } \\
\text { boom -3 } \\
\text { M }\end{array}$ & $\begin{array}{l}\text { Mosquito } \\
\text { Turbine } \\
\text { without } \\
\text { Boom 2 } \\
\text { Meters }\end{array}$ & $\begin{array}{l}\text { Mosquito } \\
\text { Turbine } \\
\text { without } \\
\text { Boom 1 } \\
\text { Meter }\end{array}$ \\
\hline $\begin{array}{l}\text { N-S } \\
\text { Permanent }\end{array}$ & 78 & -37 & -9 & 4 & 8 & 12 \\
\hline $\begin{array}{l}\text { E-W } \\
\text { Permanent }\end{array}$ & 84 & 41 & 1 & 2 & 2 & 3 \\
\hline Induced & 12 & 35 & 3 & 2 & 4 & 13 \\
\hline $\begin{array}{l}\text { Time } \\
\text { variation } \\
\text { during test }\end{array}$ & 1.7 & 1.8 & 2.8 & -2.1 & -2.3 & -3.7 \\
\hline
\end{tabular}

Table 5.1 Results of signature measurements. All values in $\mathrm{nT}$.

\subsubsection{Aircraft compensation flight and results}

In the original test plan it was proposed to do a compensation flight at an altitude of several hundred meters. However, due to concerns about the ability to autorotate with the boom this compensation flight effort was cancelled. Instead of this (a poor man's substitute) a cloverleaf around the test field was flown (Figure 5.24). Part of these data was discarded due to a battery problem.

Nevertheless standard compensation procedure with Geometrics "MagComp" software was carried out and results are presented below in tables 5.2 and 5.3. This type of 
compensation accounts for permanent, induced, and Eddy currents magnetic effects, and does not compensate for rotor blades noise or electrical currents inside the aircraft.

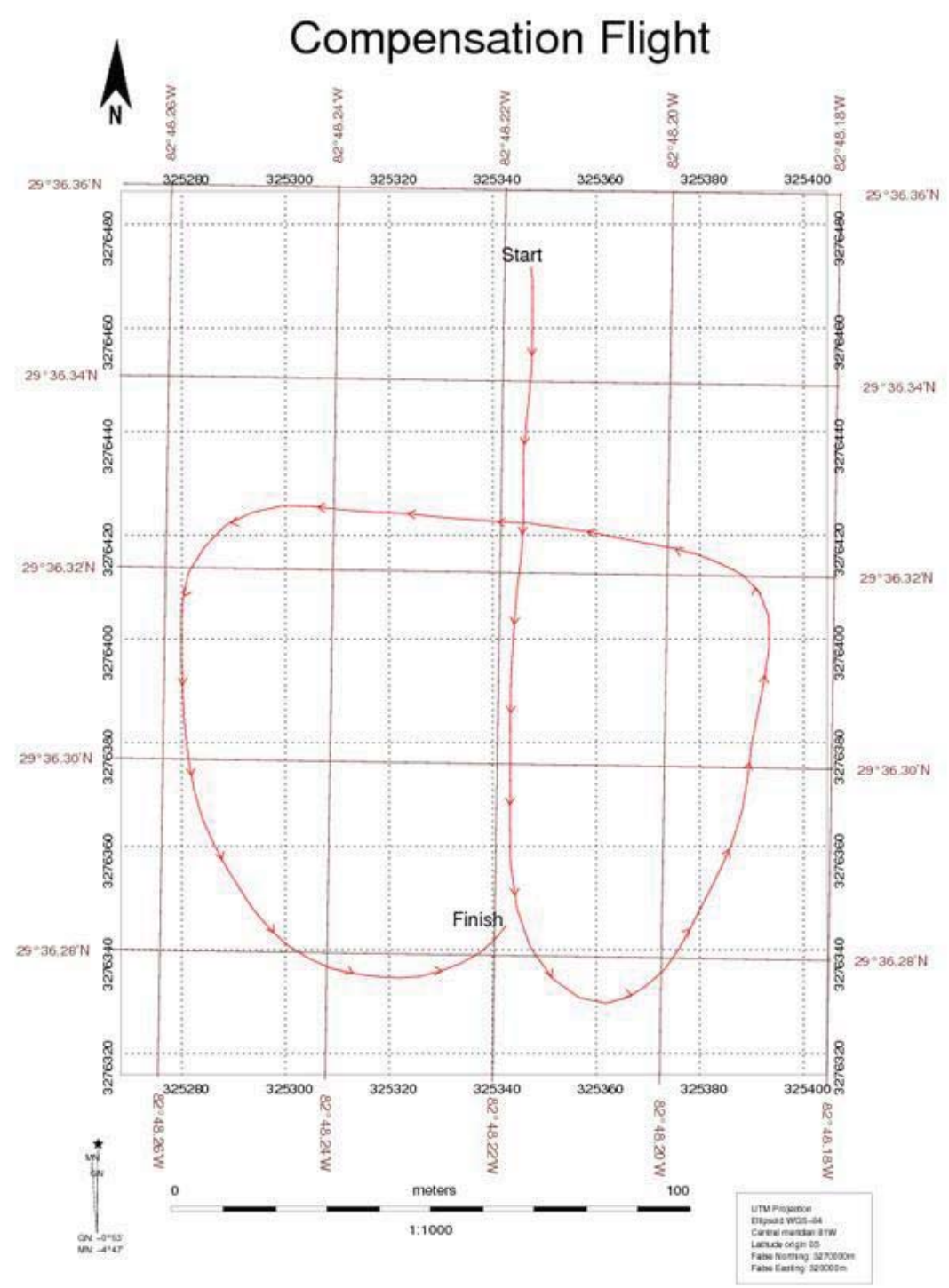

Figure 5.24 Compensation flight path 


\begin{tabular}{|c|c|c|c|c|}
\hline & Component & $\begin{array}{l}\text { Coefficient } \\
\text { Deviation }\end{array}$ & $\begin{array}{l}\text { Standard } \\
\text { maximum }\end{array}$ & $\begin{array}{l}\text { Approximate } \\
\text { Effect }\end{array}$ \\
\hline \multicolumn{5}{|l|}{$\begin{array}{l}\text { Permanent } \\
\text { Fields }\end{array}$} \\
\hline Transverse & 1 & $1.04 \mathrm{E}+01$ & $1.10 \mathrm{E}+00$ & 10.44 \\
\hline Longitudinal & 2 & $-3.56 \mathrm{E}+00$ & $8.60 \mathrm{E}-01$ & -3.56 \\
\hline Vertical & 3 & $-1.03 E+01$ & $5.40 \mathrm{E}+00$ & -10.31 \\
\hline \multicolumn{5}{|c|}{ Induced fields: } \\
\hline TT-VV & 4 & $-2.81 \mathrm{E}-01$ & $6.80 \mathrm{E}+00$ & -0.13 \\
\hline $\mathrm{LT}+\mathrm{TL}$ & 5 & $3.67 E+00$ & $4.80 \mathrm{E}-01$ & 1.73 \\
\hline $\mathrm{VT}+\mathrm{TV}$ & 6 & $-1.08 \mathrm{E}+01$ & $2.80 \mathrm{E}+00$ & -5.06 \\
\hline LL-VV & 7 & $-1.67 E+01$ & $6.80 \mathrm{E}+00$ & -7.87 \\
\hline$V L+L V$ & 8 & $9.45 \mathrm{E}+00$ & $2.30 \mathrm{E}+00$ & 4.44 \\
\hline \multicolumn{5}{|l|}{$\begin{array}{l}\text { Eddy current } \\
\text { fields }\end{array}$} \\
\hline $\mathrm{tt}-\mathrm{vv}$ & 9 & $-2.01 E+01$ & $1.20 \mathrm{E}+01$ & -1 \\
\hline It & 10 & $9.35 \mathrm{E}+00$ & $5.60 \mathrm{E}+00$ & 0.47 \\
\hline $\mathrm{vt}$ & 11 & $3.17 \mathrm{E}+01$ & $2.50 \mathrm{E}+01$ & 1.58 \\
\hline $\mathrm{tl}$ & 12 & $2.80 \mathrm{E}+01$ & $7.50 \mathrm{E}+00$ & 1.4 \\
\hline II-vv & 13 & $-1.47 E+01$ & $9.90 \mathrm{E}+00$ & -0.73 \\
\hline $\mathrm{vl}$ & 14 & $1.82 \mathrm{E}+01$ & $2.00 \mathrm{E}+01$ & 0.91 \\
\hline tv & 15 & $-5.81 E+00$ & $4.40 \mathrm{E}+00$ & -0.29 \\
\hline IV & 16 & $-8.09 E+00$ & $3.30 \mathrm{E}+00$ & -0.4 \\
\hline
\end{tabular}

Table 5.2 Compensation table for top sensor. 


\begin{tabular}{|l|r|r|r|r|}
\hline & Component & $\begin{array}{c}\text { Coefficient } \\
\text { Deviation }\end{array}$ & $\begin{array}{c}\text { Standard } \\
\text { maximum }\end{array}$ & $\begin{array}{c}\text { Approximate } \\
\text { Effect }\end{array}$ \\
\hline Permanent Fields & & & & 12.86 \\
\hline Transverse & 1 & $1.29 \mathrm{E}+01$ & $1.30 \mathrm{E}+00$ & -3.61 \\
\hline Longitudinal & 2 & $-3.61 \mathrm{E}+00$ & $1.00 \mathrm{E}+00$ & -13.69 \\
\hline Vertical & 3 & $-1.37 \mathrm{E}+01$ & $6.40 \mathrm{E}+00$ & \\
\hline & & & & \\
\hline Induced fields: & & & & -0.33 \\
\hline TT-VV & 4 & $-7.01 \mathrm{E}-01$ & $8.10 \mathrm{E}+00$ & 1.82 \\
\hline LT+TL & 5 & $3.87 \mathrm{E}+00$ & $5.70 \mathrm{E}-01$ & -7.64 \\
\hline VT+TV & 6 & $-1.63 \mathrm{E}+01$ & $3.40 \mathrm{E}+00$ & -7.77 \\
\hline LL-VV & 7 & $-1.65 \mathrm{E}+01$ & $8.10 \mathrm{E}+00$ & 5.18 \\
\hline VL+LV & 8 & $1.10 \mathrm{E}+01$ & $2.70 \mathrm{E}+00$ & \\
\hline & & & & \\
\hline Eddy current \\
fields & & & & -0.91 \\
\hline tt-vv & 9 & $-1.81 \mathrm{E}+01$ & $1.40 \mathrm{E}+01$ & 0.24 \\
\hline Lt & 10 & $4.76 \mathrm{E}+00$ & $6.70 \mathrm{E}+00$ & 1.63 \\
\hline Vt & 11 & $3.26 \mathrm{E}+01$ & $3.00 \mathrm{E}+01$ & -0.63 \\
\hline TI & 12 & $3.47 \mathrm{E}+01$ & $9.00 \mathrm{E}+00$ & 1.03 \\
\hline II-vV & 13 & $-1.26 \mathrm{E}+01$ & $1.20 \mathrm{E}+01$ & -0.3 \\
\hline VI & 14 & $2.06 \mathrm{E}+01$ & $2.40 \mathrm{E}+01$ & -0.32 \\
\hline TV & 15 & $-6.00 \mathrm{E}+00$ & $5.30 \mathrm{E}+00$ & \\
\hline Lv & 16 & $-6.40 \mathrm{E}+00$ & $3.90 \mathrm{E}+00$ & \\
\hline
\end{tabular}

Table 5.3 Compensation table for bottom sensor

It can be seen from these tables that maximum possible magnetic disturbance due to the aircraft is because of its permanent magnetization (10-15 nT), then induced effects (5$7 \mathrm{nT})$, and finally Eddy currents (1-2 nT). From the compensation graphs it can be seen that about $2 \mathrm{nT}$ noise is removed. The nature of sharp excursions recorded by bottom sensor is not known. These might be due to some wiring problems in the boom.

\subsection{Summary of field testing efforts}

The field efforts were successful in demonstrating both the feasibility of a modular approach to UAV magnetic rotorcraft as well as the feasibility of individual components of such a system. Specifically, we demonstrated the stability and ability to terrain follow of a boom equipped autonomous rotorcraft as well as the ability to collect high quality magnetic data from a small manually piloted rotorcraft which could be made autonomous. 


\section{Summary and next steps}

\subsection{Summary and recommendation}

As discussed initially in (Versteeg, McKay et al. 2007) and more in depth in this report UAV magnetometer rotorcraft systems are feasible. Such systems would be applicable to a significant percentage of DoD sites (as discussed in chapter 3), the modular components required to build such systems are available and sufficiently mature (chapter 4), and as shown by the data presented in chapter 5, if appropriate care is taken in platform selection and component integration, the performance of such systems is comparable to manual surveys. It is also clear that there is a continuum of systems which could be designed ranging from systems based on rotorcraft with $10 \mathrm{lb}$ payload (such as the Mongoose) to systems bases on rotorcraft such as the Mosquito (with $250 \mathrm{lbs}$ payload). It is also clear that no system will be ideal to all sites.

We here recommend a modular approach to system design and construction, as well as an effort in which two systems would be designed simultaneously (roughly corresponding to the two configurations described in table 3.2). These systems would share a common, open architecture and Operator Control Unit/User interface.

Our recommendation is based on the following arguments

1. It is likely that there will be significant advances in all of the components associated with an UAV magnetometer rotorcraft system. By taking a modular approach one allows organically for a model in which improved components can be integrated easily in existing systems

2. Experience has shown that the market for medium to large sized RC controllable rotorcraft is quickly evolving. A modular approach avoids the risk of locking in with a manufacturer which may go out of business or whose products may not be available. On a similar note, it is unlikely that one manufacturer provides the specific rotorcraft which would be used for all the systems.

3. An open architecture (which allows for the rapid integration of auxiliary sensors and datastreams) provides for the most flexibility in system design

In the remainder of this chapter we provide an outline of the research effort associated with developing a prototype system.

\subsection{Research effort associated with developing prototype systems}

As discussed above, given the results provided in this report as well as the information provided in (Versteeg, McKay et al. 2007) the project team feels that the logical next step would be the design, construction and testing of several modular prototype autonomous UAV helicopter-magnetometer systems. We currently feel that one of these systems 
would use the Mosquito airframe (capable of carrying a $250 \mathrm{lb}$ payload) whereas the other would use a SiCK 70 airframe (capable of carrying a $60 \mathrm{lb}$ payload). The

magnetometer configuration on both systems is to be decided as part of the design effort, but it is likely that the SiCK 70 system would carry about three magnetometers, whereas the Mosquito system would carry about eight magnetometers. Both systems would be fully autonomous in takeoff, data collection and landing. Operators would indicate which areas to survey through a map based interface and the system would take off, survey, and return for refueling. Such a system would have on board intelligence allowing for automatic grid refinement, as well as transmit data to a ground station allowing near real time target characterization and identification.

The design, construction and testing of such systems requires addressing of a range of issues, both research related as well as engineering related. Below we provide a detailed outline of the elements of our proposed effort. Note that in this outline we have not included go/no go decisions, detailed timelines, the location and details of test sites and test efforts and many other specifics which will be provided in a full proposal. However, this outline should be sufficient to provide a good insight as to what a follow-up effort would entail

A follow-up effort is expected to take about three years, and would consist of the following six high level tasks

1. Development of a detailed system requirement document (Year 1)

2. Overall architecture and software interface design and implementation (including hardware in the loop simulator and OCU) (Year 1 and 2)

3. Hardware component selection, modification and integration (Year 1 and 2)

a. Sensor package components (Year 1)

b. Rotorcraft and boom geometry (Year 1)

c. Overall system assembly (Year 1 and 2)

4. Controller enhancement, optimization and tuning (Year 1 and 2)

a. Navigation

b. Obstacle avoidance

5. Real time geophysical data processing (including development and validation of reactive airborne characterization methodology (Years 1 through 3))

6. Component and system testing efforts (Year 1 through 3)

\section{Task 1: Development of a detailed system requirement document}

Under this task we will (in discussion with the program office and broader user community) develop detailed system requirements. These will include, but not be limited to the following

1. Initial range of terrains (including the identification of prototype sites) which would be used by this system, as well as the vegetation and topographic details of 
these sites and the available data (e.g. location of powerlines and digital elevation models)

2. Operational requirements (altitude, speed, flying time, time per acre, acceptable cost per acre, operator training requirements, system reliability) which would be required by the end user for such systems to be relevant to DoD needs

3. Regulatory requirements (certification, likely operational constraints based on ES\&H rules)

4. System performance requirements, both for initial detection and potential characterization of potential UXO

This system document will be used as the blueprint for the design of the two systems and will be a deliverable three months after the start of the project

\section{Task 2: Overall architecture and software interface design and implementation (including hardware in the loop simulator)}

Under this task we will design the overall architecture of the system (hardware and software) as well as the software for all the components (Operator Control Unit, Data acquisition unit, Controller) and APIs between these components. For some of these we will base our efforts on existing software (for instance, the controller effort will extend the Guided System software package), whereas for others we will integrate commercial and open source components (for instance, we will implement a relational database for real time storage of the collected data on the ground controller) which will be able to be accessed by spatial visualization software as well as data processing software.

This task will also include the design and implementation of a "hardware in the loop simulator" which will allow detailed testing of component performance. This simulator will run the same software as the real system, but will be able to interact with a simulated rotorcraft, terrain and so on. This will allow for rapid development and testing, and should reduce (but not eliminate) the need for in field troubleshooting of the interplay between hardware and software components.

This task will also include the design of an OCU (Operator Control Unit). This will be used for system tasking, data storage, result visualization, and potentially preprocessing.

The architecture will be designed in year 1, and the components and simulator and OCU will be developed and refined in years 1 and 2 .

\section{Task 3: Hardware component selection, modification and integration}

Under task 3 (which will be initiated at the completion of task 1) we will select, test and integrate the hardware components for the system. This will be done in three steps.

Step 1: sensor selection and integration. In this step the magnetic sensors, position sensors (RTK DGPS, laser altimeter), obstacle avoidance sensors (laser, radar, optical flow), data acquisition and data transmission hardware will be selected, tested and 
integrated. As there is a synergy between the needs of the rotorcraft control system and the geophysical acquisition needs the hardware components will be integrated into one architecture (see task 2).

Step 2: selection of the rotorcraft, boom geometry, overall component mounting and wiring. In this step the final selection of rotorcraft and boom geometry and overall component mounting will be done.

Step 3: component integration. In this step all the components will be integrated on the rotorcraft. Note that this integration is likely to be done in a stepwise fashion (for instance, initially dummies may be used for the sensors to test mechanical stability), with extensive testing occurring at each step. Also note that (for resource reasons) components may be shared between rotorcraft (e.g. magnetometers, DGPS units and data acquisition hardware)

\section{Task 4: Controller enhancement, optimization and tuning (Year 1 and 2)}

This task has two components. The first is a R\&D component, in which improved algorithms will be developed and tested for navigation and obstacle avoidance. The second is an implementation component in which the controller will be integrated on the systems developed under task 3 .

\section{Task 5 Real time geophysical data processing (including development and validation of reactive airborne characterization methodology (Years 1 through 3))}

The architecture of the system will be such that the data collected by the system can be used in real time. In task 5 we will implement real time geophysical data processing both compensation of the recorded data (allowing for real time visualization and possible target inversion) and as input for an adaptive acquisition effort. Specifically, one of the objectives is to have this system be able to collect high quality data which can be used for discrimination purposes. For this, our current notion is to implement a trigger based on magnetic gradient change to go in characterization mode. In this mode the system would hover over a target area and collect high quality data which could be used for enhanced characterization purposes. This characterization mode could be accompanied by the collection of image data, allowing the collection of a dataset which would allow operators to make more informed decisions on the need for subsequent land based acquisition efforts.

\section{Task 6 Component and system testing efforts (Year 1 through 3)}

Under task 6 all the component and system testing would occur. This would be done through a comprehensive test plan which would characterize all elements of the system. These tests would be done at a range of locations (including the INL site, which provides for an ideal environment in which to test system performance over large areas). 


\title{
7 Appendix 1: contact information
}

\author{
Roelof Versteeg \\ Idaho National Laboratory \\ PO Box 1625 \\ Idaho Falls, ID \\ Phone: 2085264437 \\ Fax: 2085262639 \\ Cell: $\quad 2085691606$ \\ email: roelof.versteeg@inl.gov
}

\author{
Mark McKay \\ Idaho National Laboratory \\ PO Box 1625 \\ Idaho Falls, ID \\ Phone: 2085260539 \\ Fax: 2085267688 \\ email: Mark.McKay@inl.gov
}

\author{
Matt Anderson \\ Idaho National Laboratory \\ PO Box 1625 \\ Idaho Falls, ID \\ Phone: 2085264308 \\ Fax: 2085267688 \\ email: Matthew.Anderson@inl.gov
}

Ross Johnson

Geometrics, Inc

2190 Fortune Dr.

San Jose, Ca. 95131 U.S.A.

Phone: 408-428-4242

Cell: 408-515-0266

Fax: 408-954-0902

Email: ross@geometrics.com

Darryl Curley

Geometrics, Inc 
2190 Fortune Dr.

San Jose, Ca. 95131 U.S.A.

Phone: 408-835-9576

Cell: 408-835-9576

Fax: 408-954-0902

Email: dcurley@geometrics.com

Les Beard

Battelle, Inc

BeardL@battelle.org

Phone: 865-483-2541

Cell: $865-804-8662$

Dwight Junkin

Composite FX

dwight@composite-fx.com

Phone: (352) 5381624

Scott Vinson

Guided Systems

sv@guidedsys.com

770-898-9100 ext 878

Eric Corban

Guided Systems

ec@guidedsys.com

770-898-9100 ext 832

Mikhail Tchernychev

Geometrics, Inc

2190 Fortune Dr.

San Jose, Ca. 95131 U.S.A.

Fax: 408-954-0902

misha@mail.geometrics.com 


\section{References}

Billings, S. and Wright, D. (2009), Next generation Helimag UXO Mapping Technology Demonstration Report, Final report for ESTCP Project MM-0471.

Chapuis, J., C. Eck, H. P. Geering and H. Allenspach (1997). Autonomously Flying Helicopter. 4th IFAC Symposium on Advances in Control Education, Istanbul, Turkey.

Doll, W. E., T. J. Gamey and J. S. Holladay (2001). Current Research into Airborne UXO Detection. SAGEEP Annual Meeting, Oakland, CA.

Gamey, T. J., W. E. Doll, L. P. Beard and D. T. Bell (2003). Analysis of noise coherence in airborne magnetic gradients for UXO detection. 2003 SAGEEP Meeting, San Antonio, TX.

Gamey, T. J. and R. Mahler (1999). A Comparison of Towed and Mounted Helicopter Magnetometer Sys tems for UXO Detection. SAGEEP 1999, EEGS.

MIT (1998). MIT Entry in the 1998 International Aerial Robotics Competition. IARC, Hanford, WA.

Nelson, H. H., D. L. Wright, T. Furuya, J. R. McDonald, N. Khadr and D. A. Steinhurst (2004). MTADS Airborne and Vehicular Survey of Target S1 at Isleta Pueblo, Albuquerque, NM, 17 February - 2 March 2003, Naval Research Laboratory.

Salem, A., T. J. Gamey, D. Ravat and K. Ushijima (2001). Automatic Detection of UXO from Airborne Magnetic Data using a Neural Network. Proceedings of the 2001 Symposium on the Application of Geophysics to Engineering and Environmental Problems, Denver.

Stanley, J. M. (2003). Approaching 100\% Quality Assurance in Ferrous Explosive Ordnance Disposal. Armidale, Australia, Geophysical Technology Limited: 10.

Versteeg, R., M. McKay, M. Anderson, R. Johnson, B. Selfridge and J. Bennett (2007). Feasibility study for an Autonomous UAV -Magnetometer system, Idaho National Laboratory - INL/EXT-07-13386 55 pages. 